\title{
Exact Resummations in the Theory of Hydrodynamic Turbulence: I. The Ball of Locality and Normal Scaling
}

\author{
Victor L'vovi and Itamar Procaciaid

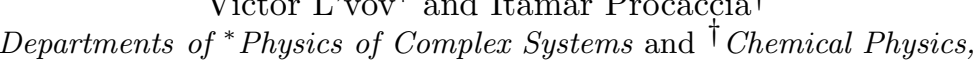 \\ The Weizmann Institute of Science, Rehovot 76100, Israel, \\ Institute of Automation and Electrometry, Ac. Sci. of Russia, 630090, Novosibirsk, Russia
}

\begin{abstract}
This paper is the first in a series of three papers that aim at understanding the scaling behaviour of hydrodynamic turbulence. We present in this paper a perturbative theory for the structure functions and the response functions of the hydrodynamic velocity field in real space and time. Starting from the Navier-Stokes equations (at high Reynolds number Re) we show that the standard perturbative expansions that suffer from infra-red divergences can be exactly resummed using the Belinicher-L'vov transformation. After this exact (partial) resummation it is proven that the resulting perturbation theory is free of divergences, both in large and in small spatial separations. The hydrodynamic response and the correlations have contributions that arise from mediated interactions which take place at some space- time coordinates. It is shown that the main contribution arises when these coordinates lie within a shell of a "ball of locality" that is defined and discussed. We argue that the real space-time formalism developed here offers a clear and intuitive understanding of every diagram in the theory, and of every element in the diagrams. One major consequence of this theory is that none of the familiar perturbative mechanisms may ruin the classical Kolmogorov (K41) scaling solution for the structure functions. Accordingly, corrections to the K41 solutions should be sought in nonperturbative effects. These effects are the subjects of papers II and III in this series, that will propose a mechanism for anomalous scaling in turbulence, which in particular allows multiscaling of the structure functions.
\end{abstract}

PACS numbers 47.27.Gs, 47.27.Jv, 05.40.+j

\section{INTRODUCTION TO THE SERIES OF PAPERS}

This paper is the first in a series of three papers that aim for a systematic elucidation of the analytic structure of the the statistical theory of hydrodynamic turbulence at high Reynolds number Re. The natural quantities that appear in the statistical description of turbulence are averages of products of hydrodynamic fields at different space-time points. The aim of the analytic approach is to evaluate such quantities on the basis of the equations of fluid mechanics.

The fundamental field in hydrodynamics is the velocity field of the fluid, denoted as $\mathbf{u}(\mathbf{r}, t)$ where $\mathbf{r}$ is a point in $d$-dimensional space (usually $d=2$ or 3 ) and $t$ is the time. Statistical quantities that attracted years of experimental and theoretical attention [1,2, 2,3] are the structure functions of velocity differences, denoted as $S_{n}(R)$

$$
S_{n}(R)=\left\langle|\mathbf{u}(\mathbf{r}+\mathbf{R}, t)-\mathbf{u}(\mathbf{r}, t)|^{n}\right\rangle .
$$

where $\langle\ldots\rangle$ stands for a suitably defined ensemble average. It has been stipulated for a long time that the structure functions scale as a function of $R$ when $R$ is in the so called "inertial range", i.e. $\eta \ll R \ll L$ with $\eta$ being the inner viscous scale and $L$ being the outer integral scale of turbulence:

$$
S_{n}(R) \sim R^{\zeta_{n}}, \quad \eta \ll R \ll L
$$

with $\zeta_{n}$ being scaling exponents. One of the major questions in fundamental turbulence research is whether these scaling exponents are correctly predicted by the classical Kolmogorov 41 theory in which $\zeta_{n}=n / 3$, or whether these exponents manifest the phenomenon of "multiscaling" with $\zeta_{n}$ being a nonlinear function of $n$, as has been indicated by experiments.

Experimental research did not confine itself to the measurement of the structure functions of velocity differences. Gradient fields featured as well. For example the correlation function of the energy dissipation field has been studied extensively. The dissipation field $\epsilon(\mathbf{r}, t)$ is defined as

$$
\epsilon(\mathbf{r}, t) \equiv \frac{\nu}{2}\left[\nabla_{\alpha} u_{\beta}(\mathbf{r}, t)+\nabla_{\beta} u_{\alpha}(\mathbf{r}, t)\right]^{2}
$$

with $\nu$ being the kinematic viscosity. The correlation function of the dissipation field $K_{\epsilon \epsilon}(R)$ is

$$
K_{\epsilon \epsilon}(R)=\langle\hat{\epsilon}(\mathbf{r}+\mathbf{R}, t) \hat{\epsilon}(\mathbf{r}, t)\rangle,
$$

where $\hat{\epsilon}(\mathbf{r}, t)=\epsilon(\mathbf{r}, t)-\bar{\epsilon}$. Here and below $\bar{\epsilon} \equiv\langle\epsilon\rangle$. It was claimed experimentally [1,2, 3 that $K_{\epsilon \epsilon}(R)$ decays as a power law,

$$
K_{\epsilon \epsilon}(R) \sim R^{-\mu}, \quad \eta \ll R \ll L
$$

with $\mu$ having a numerical value of $0.2-0.3$. The analytic derivation of this law from the equations of fluid mechanics, and the calculation of the numerical value of 
the scaling exponent $\mu$ have also been elusive goals of the theoretical research.

In the present series of three papers we will present a systematic theory that culminates in the analytic estimate of the scaling exponents $\zeta_{n}$ and $\mu$, in addition to the calculation of other quantities of experimental and theoretical interest. Since the road to these results is not straightforward, we present here a summary of the three papers in the series, hoping to motivate the reader to examine the necessary steps of analysis even though they are somewhat tedious. We believe that the proposed structure of the resulting scaling theory is rather beautiful and simple, justifying in part the lengthy path taken to reach it.

The present paper which is numbered I deals with the perturbative theory of the correlation, response and structure functions of hydrodynamic turbulence. The main result of this paper (and see also 44) is that after appropriate resummations and renormalizations the perturbative theory for these quantities is finite order by order in the sense that all the integrals appearing in the theory are convergent in the ultraviolet and the infrared limits. The meaning of this result is that there is no perturbative mechanism to introduce a length scale into the theory of the structure functions. When the perturbative series diverges either in the infrared or in the ultraviolet the cutoff lengths that are used to tame the divergence serve as renormalization scales which can be used to change the scaling exponents from the prediction of dimensional analysis. This result indicates that as far as the perturbative theory is concerned there is no mechanism to correct the exponents $\zeta_{n}$ from their K41 values. Of course, nonperturbative effects can very well furnish such a mechanism and therefore in paper II [5] (subtitled "A Ladder to Anomalous Scaling) we turn to the analysis of nonperturbative effects. There is more than one source of nonperturbative effects that need to be studied. One is obtained from summing up infinite perturbative series into analytic equations that display solutions that are not obtainable from the initial perturbation series. Another one stems from sum rules or constraints on the perturbation theory as a whole. In paper II we study infinite resummations of ladder type diagrams. It is shown there that the renormalized perturbation theory for correlation functions that include velocity derivatives (to second or higher power) exhibit in their perturbation expansion a logarithmic dependence on the viscous scale $\eta[7,8]$. In this way the inner scale of turbulence appears explicitly in the analytic theory. The perturbative series can be resummed to obtain integrodifferential equations for some many-point objects of the theory. These equations have also non-perturbative scale-invariant solutions that can be represented as power laws of $\eta$ to some exponents $\Delta$. For example it will be shown in paper III [6] subtitled (Scenarios for Anomalous Scaling and Intermittency) that the correlation function of the energy dissipation field has such dependence:

$$
K_{\epsilon \epsilon}(R) \sim \bar{\epsilon}^{2}(L / R)^{2 \zeta_{2}-\zeta_{4}}(R / \eta)^{2\left(\Delta-\Delta_{c}\right)}
$$

where $\Delta_{c}=2-\zeta_{2}$. It has been argued [9] that if $\Delta<\Delta_{c}$ (a situation referred to as the "subcritical scenario"), then K41 is asymptotically exact for infinite Re. Then $2 \zeta_{2}=\zeta_{4}=4 / 3$ and the outer scale $L$ disappears from (1.6). In that case the exponent $\mu$ is identified with $2(4 / 3-\Delta)$, and the renormalization length is the inner length $\eta$. In fact, in paper II it will be shown that the exponent $\Delta$ can be computed exactly, and that it takes on exactly the critical value $\Delta=\Delta_{c}$. As a result of this the correlation $K_{\epsilon \epsilon}(R)$ can be shown to depend on $R$ like

$$
K_{\epsilon \epsilon}(R) \simeq \bar{\epsilon}^{2}(R / L)^{\zeta_{4}-2 \zeta_{2}}
$$

In other words, the critical situation $\Delta=\Delta_{c}$ results in the disappearance of the inner renormalization scale and the appearance of the outer renormalization scale in (1.7). In addition one notes that $K_{\epsilon \epsilon}(R)$ decays as a function of $R$ (i.e. the correlation is mixing) only if $\zeta_{4}<2 \zeta_{2}$ which implies deviations from K41. Thus we will argue in paper II that the critical scenario $\Delta=\Delta_{c}$ goes hand in hand with multiscaling if $K_{\epsilon \epsilon}(R)$ is mixing and then $\mu$ is identified with $\zeta_{4}-2 \zeta_{2}$. Paper III which is the last paper in this series focuses on the analysis of the nonperturbative constraint furnished by the ballance equation which is obtained from the equations of motion of the structure functions. This analysis exposes a mechanism for multiscaling which leads to the evaluation of the scaling exponents $\zeta_{n}$ from first principles.

\section{ON THE BACKGROUND OF THE ANALYTIC DESCRIPTION OF TURBULENCE}

Of foremost importance in the analytical approach have been those methods that rely on some perturbative treatment of the Navier-Stokes equation 110,11, 12, 13, 14, , 4 . Such treatments are fraught with difficulties, which stem basically from two sources. The main source is the fundamental fact that turbulence has no small parameter. Thus, any naive perturbation scheme is bound to fail, being effectively a non-convergent series in powers of Re. Any truncation of such a series leads to disastrous nonsense. Evidently one must cleverly resum such series to obtain a formal perturbation expansion in terms of an effective coupling constant which differs from Re. The second source of difficulty is that the natural statistical objects which appear in the perturbation expansions [10,11, 13, 14] of the Navier-Stokes equation are correlation functions $\mathcal{F}_{\alpha \beta}\left(\mathbf{r}, \mathbf{r}^{\prime}, t, t^{\prime}\right)$ of the velocity field $\mathbf{u}(\mathbf{r}, t)$ itself:

$$
\mathcal{F}_{\alpha \beta}\left(\mathbf{r}, \mathbf{r}^{\prime}, t, t^{\prime}\right)=\left\langle u_{\alpha}(\mathbf{r}, t) u_{\beta}\left(\mathbf{r}^{\prime}, t^{\prime}\right)\right\rangle
$$

The problem is that the correlator $F_{\alpha \beta}\left(\mathbf{r}, \mathbf{r}^{\prime}, t, t^{\prime}\right)$ is not universal, since it is dominated by contributions to $u_{\alpha}(\mathbf{r}, t)$ which come from the largest scales in the fluid flow which are determined by the features of the energy 
injection mechanisms. This physical fact is reflected in the theory as infra-red divergences that have plagued the discussion of the analytic approach for decades. Indeed, all the early attempts to develop a consistent analytic approach to turbulence, notably the well known theories of Wyld [10] and of Martin, Siggia and Rose [11], share this problem.

It is ironic that the appropriate universal objects were known all along due to the insight of Richardson 16, 17 and later of Kolmogorov [18], who realized that velocity differences across a lengthscale $R$ do possess good properties, since they are dominated by motions of scale $R$. In Kolmogorov's language such a velocity difference is expected to be universal when $R$ is in "inertial range". It seems advisable to develop the analytic techniques also in terms of similar universal objects. To this aim one may transform the equations of motion to new variables. A potentially promising approach could be to recast the Navier-Stokes equation in terms of Lagrangian coordinates as has been attempted by Kraichnan [12]. The equation for the Lagrangian velocity involves however the Eulerian velocity which relates to the Lagrangian velocity via a complicated time evolution operator. A lot has been accomplished in this approach; firstly, Kraichnan acheived a qualitative undertsanding of the different type of power law spectra that appear in different contexts like scalar pasive diffusion, two and three dimensional turbulence, and Burgers dynamics. Secnodly, a numerical prediction for the Kolmogorov constant was obtained. However, the resulting perturbation theory does not have a diagrammatic representation in the sense that one does not know how to achieve infinite resummations. The progress in understanding non-perturbative aspects remained unsatisfactory.

Another approach that claimed success was due to Belinicher and L'vov [4] who introduced an exact transformation to variables which they called "quasiLagrangian". This term was not particularly successful; on the one hand it could give the impression of an approximate scheme, and on the other hand it implied proximity to the Lagrangian transformation. Both impressions are incorrect. The Belinicher-L'vov transformation is exact, and it has very little to do with the cumbersome features of the Lagrangian transformation. In terms of the Eulerian velocity $\mathbf{u}(\mathbf{r}, t)$ they defined the field $\mathbf{v}\left(\mathbf{r}_{0}, t_{0} \mid \mathbf{r}, t\right)$ as

$$
\mathbf{v}\left(\mathbf{r}_{0}, t_{0} \mid \mathbf{r}, t\right) \equiv \mathbf{u}\left[\mathbf{r}+\boldsymbol{\rho}\left(\mathbf{r}_{0}, t\right), t\right]
$$

where

$$
\boldsymbol{\rho}\left(\mathbf{r}_{0}, t\right)=\int_{t_{0}}^{t} \mathbf{u}\left[\mathbf{r}_{0}+\boldsymbol{\rho}\left(\mathbf{r}_{0}, \tau\right), \tau\right] .
$$

Note that $\boldsymbol{\rho}\left(\mathbf{r}_{0}, t\right)$ is precisely the Lagrangian trajectory of a fluid particle that is positioned at $\mathbf{r}_{0}$ at time $t=t_{0}$. Nevertheless the field $\mathbf{v}\left(\mathbf{r}_{0}, t_{0} \mid \mathbf{r}, t\right)$ becomes in time very different from the Lagrangian field in which the Lagrangian trajectories of all space points are involved.
The observation of Belinicher and L'vov was that the variables $\mathbf{w}\left(\mathbf{r}_{0}, t_{0} \mid \mathbf{r}, t\right)$ which are defined as

$$
\mathbf{w}\left(\mathbf{r}_{0}, t_{0} \mid \mathbf{r}, \mathbf{t}\right) \equiv \mathbf{v}\left(\mathbf{r}_{0}, t_{0} \mid \mathbf{r}, t\right)-\mathbf{v}\left(\mathbf{r}_{0}, t_{0} \mid \mathbf{r}_{0}, t\right)
$$

exactly satisfy the Navier-Stokes equation in the limit of incompressible fluid. Consequently they could develop a diagrammatic perturbation theory in terms of these variables. The resulting theory was free of the two related problems that we discussed above: the $\langle\mathbf{w w}\rangle$ correlators were universal for $\left|\mathbf{r}-\mathbf{r}_{0}\right|$ in the inertial range, and the theory was free of infra-red (and ultra-violet) divergences. They also showed that the Kolmogorov 1941 scaling is an order by order solution of the resulting theory. Two important properties of this formulation are: (i) The simultaneous correlators of $\mathbf{v}\left[\mathbf{r}_{0} \mid \mathbf{r}, t\right]$ are identical to the simultaneous correlators of $\mathbf{u}(\mathbf{r}, t)$. The reason is that for stationary statistics the simultaneous correlators of an arbitrary number of factors of $\mathbf{v}\left[\mathbf{r}_{0} \mid \mathbf{r}, t\right]$ does not depend on $t$, and in particular one can take $t=t_{0}$. The property then follows directly from Eq.(2.3). (ii) The correlators of $\mathbf{w}$ are closely related to the structure functions of $\mathbf{u}(1.1)$. Clearly

$$
S_{n}\left(\left|\mathbf{r}-\mathbf{r}_{0}\right|\right)=\left\langle\left|\mathbf{w}\left(\mathbf{r}_{0}, t_{0} \mid \mathbf{r}, t\right)\right|^{n}\right\rangle .
$$

As the structure functions of the Eulerian velocity differences were for years at the focus of experimental research, the formulation in terms of the variables $\mathbf{w}$ gives a direct link between theory and experiments. We will refer to the variable $\mathbf{w}\left(\mathbf{r}_{0}, t_{0} \mid \mathbf{r}, t\right)$ as the $\mathrm{BL}$ velocity differences.

For some reason these results have not been widely accepted. One reason must have been the fact that the theory involved the subtraction of a velocity field at one special point (denoted $\mathbf{r}_{0}$ above), and this, in the words of Kraichnan [19], "cannot cure the infra red divergences that stem from far away regions". Possibly the main reason for disbelief is that the Kolmogorov solution was found to be an exact order by order solution of the theory. There are two reasons not to believe a theory that reproduces K41. Experimentally, it was widely stated that observations of the structure functions of velocity differences are in contradiction with the K41 scaling, see for example [20,21] and references therein. Theoretically, it was believed since the days of Landau that the strong dissipation fluctuations "must" renormalize the exponents that are found within "naive" dimensional analysis (as is commonly found in the context of critical phenomena etc.). Lastly, the issue of the vertex renormalization was left out in [4] for future work. This also gave reasons to doubt whether the demonstration of the K41 scaling solution is to be accepted. The wisdom of the community about this issue was summarized recently in one sentence 22]: "The quasi- Lagrangian formulation serves only to provide a sophisticated diagrammatic reformulation of the Kolmogorov argument".

The aim of this paper is to clarify and solidify the theoretical points that are needed to construct a Galileaninvariant and divergence-free renormalized perturbation 
scheme for turbulence, and to answer all the doubts described above. Our strategy is to develop the theory from the start entirely in $\mathbf{r}, t$ representation in terms of new propagators which are correlations and Green's functions of the BL velocity differences (2.4); these objects possess the good properties of being universal and of having transparent physical significance. We find that this new approach enjoys the merit that each diagram and the elements of the diagrams have definite physical interpretation. The simultaneous correlation functions of our theory are the functions of the Eulerian velocity differences. We shall see that the physical reason for the lack of infra-red divergences is the local character of the hydrodynamic interactions. This character is reflected in the fast decay of the Green's functions when one of its spatial arguments goes away from a "ball of locality" whose radius is computed in the theory. The intuitive idea of the locality of interaction in fluids in $\mathbf{r}$ space finds an immediate reflection in the structure of the theory. Only interactions inside the ball of locality turn out to be important.

As a result of this theory we will explain why the K41 scaling is indeed a consistent order by order solution of the theory. Notwithstanding, we will argue in papers II and III of this series that nonperturbative effects may furnish a multiscaling solution which differs from K41. The essence of the theory is that such a solution cannot be seen in order by order expansions.

The structure of this paper is as follows: In Section 2 we present the equations of motion, introduce the appropriate variables, and derive, via the path integral formulation, the Dyson-Wyld equations for the second order correlators and Green's functions (i.e the propagators). In Section 3 we discuss the physical meaning of the propagators and of the mass operators in our formulation, study the properties of the propagators, and show how they depend on time and on the coordinates. The crucial property of the Green's function in BL variables is that its bare value is given as the difference between two projection operators. This serves as an initial value for the equation of motion of our Green's function, leading to solutions that differ fundamentally from the Green's function in the Eulerian representation. The properties discovered in Section 3 allow us to present in Section 4 the proof of locality. This is the central section of this paper; it is shown that all the diagrams that appear in the infinite series for the mass operators in the Dyson-Wyld equations are local. This means that they have no divergences either in the large space- time separation limit or in the small separation limit, and they all contribute mostly in the "ball of locality" that is defined in section 3. As a result we will be able to show that the only consistent scaling solution (order by order) has the K41 scaling exponents. Of course, this approach suffers from the usual problem that although the series converges order by order, it may diverge as a whole. This is the possible source of nonperturbative effects that are being discussed in papers II and III of this series. Section 5 is reserved for a summary and a discussion.

\section{EQUATIONS OF MOTION, VARIABLES AND RENORMALIZATIONS}

\section{A. Equations of motion}

The analytic theory of turbulence is based on the Navier-Stokes equation for the Eulerian velocity field $\mathbf{u}(\mathbf{r}, t)$. In the case of an incompressible fluid they read

$$
\partial \mathbf{u} / \partial t+(\mathbf{u} \cdot \nabla) \mathbf{u}-\nu \nabla^{2} \mathbf{u}+\nabla p=\mathbf{f}, \quad \nabla \cdot \mathbf{u}=0
$$

where $\nu$ is the kinematic viscosity, $p$ is the pressure, and $\mathbf{f}$ is some forcing which maintains the flow. Since we are interested in incompressible flows, we project the longitudinal components out of the equations of motion. This is done with the help of the projection operator $\stackrel{\leftrightarrow}{\mathbf{P}}$ which

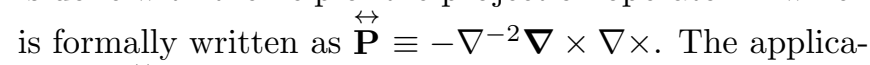
tion of $\stackrel{\leftrightarrow}{\mathbf{P}}$ to any given vector field $\mathbf{a}(\mathbf{r})$ is non local, and it has the form:

$$
[\overleftrightarrow{\mathbf{P}} \mathbf{a}(\mathbf{r})]_{\alpha}=\int d \mathbf{r}^{\prime} P_{\alpha \beta}\left(\mathbf{r}-\mathbf{r}^{\prime}\right) a_{\beta}\left(\mathbf{r}^{\prime}\right)
$$

where $P_{\alpha \beta}\left(\mathbf{r}-\mathbf{r}^{\prime}\right)$ is the inverse Fourier transform in $\mathbf{k}$ of the tensor $P_{\alpha \beta}(\mathbf{k})$ :

$$
\begin{aligned}
P_{\alpha \beta}\left(\mathbf{r}-\mathbf{r}^{\prime}\right) & =\int \frac{d^{3} k}{(2 \pi)^{3}} \exp \left[-i\left(\mathbf{r}-\mathbf{r}^{\prime}\right) \cdot \mathbf{k}\right] P_{\alpha \beta}(\mathbf{k}), \\
P_{\alpha \beta}(\mathbf{k}) & =\delta_{\alpha \beta}-\frac{1}{k^{2}} k_{\alpha} k_{\beta} .
\end{aligned}
$$

The calculation of the Fourier integral (3.3) gives

$$
\begin{aligned}
P_{\alpha \beta}\left(\mathbf{r}-\mathbf{r}^{\prime}\right) & =\delta_{\alpha \beta} \delta\left(\mathbf{r}-\mathbf{r}^{\prime}\right) \\
& -\frac{1}{4 \pi}\left[\frac{\delta_{\alpha \beta}}{\left|\mathbf{r}-\mathbf{r}^{\prime}\right|^{3}}-3 \frac{\left(r_{\alpha}-r_{\alpha}^{\prime}\right)\left(r_{\beta}-r_{\beta}^{\prime}\right)}{\left|\mathbf{r}-\mathbf{r}^{\prime}\right|^{5}}\right] .
\end{aligned}
$$

Applying $\stackrel{\leftrightarrow}{\mathbf{P}}$ to Eq. 3.1 we find

$$
\left(\partial / \partial t-\nu \nabla^{2}\right) \mathbf{u}+\overleftrightarrow{\mathbf{P}}(\mathbf{u} \cdot \nabla) \mathbf{u}=\overleftrightarrow{\mathbf{P}} \mathbf{f}
$$

We now perform the Belinicher-L'vov change of variables (2.2 2.3) together with $\mathbf{f}\left[\mathbf{r}+\boldsymbol{\rho}\left(\mathbf{r}_{0}, t_{0}, t\right), t\right]=\tilde{\boldsymbol{\phi}}\left[\mathbf{r}_{0}, t_{0} \mid \mathbf{r}, t\right]$. Using the Navier Stokes equation and the chain rule of differentiation we find the equation of motion for $\mathbf{v}\left[\mathbf{r}_{0}, t_{0} \mid \mathbf{r}, t\right]$ :

$$
\begin{aligned}
\frac{\partial}{\partial t} \mathbf{v}\left[\mathbf{r}_{0}, t_{0} \mid \mathbf{r}, t\right] & +\stackrel{\leftrightarrow}{\mathbf{P}}\left[\mathbf{w}\left[\mathbf{r}_{0}, t_{0} \mid \mathbf{r}, t\right] \cdot \nabla\right] \mathbf{w}\left[\mathbf{r}_{0}, t_{0} \mid \mathbf{r}, t\right] \\
& =\nu\left[\nabla_{0}^{2}+\nabla^{2}\right] \mathbf{w}\left[\mathbf{r}_{0}, t_{0} \mid \mathbf{r}, t\right]+\stackrel{\leftrightarrow}{\mathbf{P}} \boldsymbol{\phi}
\end{aligned}
$$

where $\mathbf{w}\left(\mathbf{r}_{0}, t_{0} \mid \mathbf{r}, t\right)$ is the BL velocity difference defined in (2.4) and $\nabla_{0}^{2}$ acts on the $\mathbf{r}_{0}$ variable. We note that 
the equation of motion is independent of $t_{0}$, and from now on we drop the argument $t_{0}$ in $\mathbf{v}\left(\mathbf{r}_{0} \mid \mathbf{r}, t\right)$. Our aim is to develop a theory in terms of the BL field $\mathbf{w}$. It can be seen from (3.7) that $\mathbf{w}$ satisfies the Navier-Stokes equation (with a different forcing). However we will use the mixed equation (3.7) since the change of variables from $\mathbf{u}$ to $\mathbf{v}$ is more direct.

\section{B. Generating functional and perturbation theory}

The development of perturbation theory can be done using a number of equivalent formalisms, such as the Wyld diagrammatics [10] or the path integral formulation 11, 13, 14, 26, 15. Both approaches have been widely reviewed, and can be shown to give identically the same diagrams [15. We follow here the approach of Ref. 13, 14, 15 which is based on the generating functional $Z(\lambda, \mathbf{m})$

$$
\begin{aligned}
Z(\boldsymbol{\lambda}, \mathbf{m})= & \frac{1}{Z} \int D \mathbf{u}(x) D \mathbf{p}(x) \exp \{i I \\
& \left.+\int d x[\mathbf{u}(x) \cdot \boldsymbol{\lambda}(x)+\mathbf{p}(x) \cdot \mathbf{m}(x)]\right\}
\end{aligned}
$$

where $x \equiv(\mathbf{r}, t)$. The quantity $I$ is referred to as the effective action. It is customary to divide $I$ into two parts, one quadratic and the other triadic in the field $\mathbf{p}$ and $\mathbf{u}$ :

$$
\begin{aligned}
I= & I_{0}+I_{\text {int }} \\
I_{0}= & \int d x\left[p_{\alpha} \frac{\partial u_{\alpha}}{\partial t}-\nu p_{\alpha} \nabla^{2} u_{\alpha}\right] \\
& +\frac{i}{2} \int p_{\alpha}(x) D_{\alpha \beta}(x-y) p_{\beta}(y) d x d y, \\
I_{\text {int }}= & \int d x p_{\alpha}(x) u_{\beta}(x) \nabla_{\beta} u_{\alpha}(x) .
\end{aligned}
$$

Here $D_{\alpha \beta}(x-y)$ is the correlation function of a Gaussian random force $\mathbf{f}$. We will not work in these variables, but rather make a change of variables in (3.8-3.11) according to (2.2 2.4 and (3.7). We will denote the resulting function as $\mathcal{Z}(\mathbf{l}, \mathbf{m})$ :

$$
\begin{aligned}
\mathcal{Z}(\mathbf{l}, \mathbf{m})= & \frac{1}{Z} \int D \mathbf{v} D \mathbf{p} \exp \{i I \\
& \left.\left.+\int d x \mathbf{v}\left(\mathbf{r}_{0} \mid x\right) \cdot \mathbf{l}(x)+\mathbf{p}(x) \cdot \mathbf{m}(x)\right]\right\}
\end{aligned}
$$

In the new variables the effective action can be again divided as in (3.9), but with

$$
\begin{aligned}
I_{0}= & \int d x\left[p_{\alpha} \frac{\partial v_{\alpha}}{\partial t}-\nu p_{\alpha} \nabla^{2} w_{\alpha}\right] \\
& +\frac{i}{2} \int p_{\alpha}(x) \mathcal{D}_{\alpha \beta}(x-y) p_{\beta}(y) d x d y, \\
I_{\text {int }}= & \int d x p_{\alpha}(x) w_{\beta}(x) \nabla_{\beta} w_{\alpha}(x) .
\end{aligned}
$$

Here $\mathcal{D}_{\alpha \beta}(x-y)$ is the correlation function of the random force $\phi$ in Eq. 3.7) which is again assumed to be Gaussian (note that the initial force $\mathrm{f}$ is not Gaussian now, but this is of no consequence). In this formalism $\int D \mathbf{v} D \mathbf{p}$ is a functional integral over all possible realizations of the incompressible velocity field $\mathbf{v}$ and the incompressible auxiliary vector field $\mathbf{p}$ at all points of space and time. Note that in this formalism the auxiliary field $\mathbf{p}$ multiplies the equation of motion for $\mathbf{v}$ and that because of the restriction of incompressibility we do not have to display the transverse projector in the effective action. The quantity $Z$ in Eq.(3.12) is a normalization constant,

$$
Z=\int D \mathbf{v} D \mathbf{p} \exp \{i I\}
$$

which is equal to 1 . The proof of this [26] is based on the expansion of the $\exp \{i I\}$. The first term of this expansion is 1 . The diagrammatic representation of the next terms will have closed loops made of Green's functions; all these diagrams are zero because of causality. Upon expanding in powers of $I_{\text {int }}$ we get from (3.12) a theory that contains summations of infinite terms compared to the theory with $\mathbf{u}(\mathbf{r}, t)$ as the variable in the path integral. To see this note that (2.2), 2.3) can be rewritten as

$$
\mathbf{u}(\mathbf{r}, t)=\mathbf{v}\left(\mathbf{r}_{0} \mid \mathbf{r}-\int_{t_{0}}^{t} \mathbf{v}\left(\mathbf{r}_{0} \mid \mathbf{r}_{0}, \tau\right) d \tau, t\right) .
$$

The RHS of this equation can be expanded in a Taylor series. The inversion of this series for $\mathbf{v}$ in terms of $\mathbf{u}$ is again an infinite series. We thus see that given powers of $\mathbf{v}$ contain infinite summations in terms of powers of $\mathbf{u}$, and vice versa. It is extremely difficult to find the explicit diagrammatic resummation of the series in $\mathbf{u}$ to get the series in $\mathbf{v}$. It is a great advantage of the path integral formulation that such infinite resummations can be obtained by a simple change of variables. This resummation is referred here as the Belinicher-L'vov renormalization. We reiterate that this renormalization is an exact formal step, and its usefulness needs to be demonstrated. It is already obvious however that although the original functional (3.8) produces correlators which are not Galilean invariant, the new one $(3.12)$ is free of this problem. This guarantees that the theory that is derived on the basis of this functional is Galilean invariant.

An average of any product of the fields $\mathbf{w}$ and $\mathbf{p}$ is computed as a functional derivative of $\mathcal{Z}(\mathbf{l}, \mathbf{m})$ with respect to $\mathbf{l}$ and $\mathbf{m}$ in the appropriate order of differentiation, finally taking the limit $l, m \rightarrow 0$. For example

$$
\begin{aligned}
F_{\alpha \beta}\left(\mathbf{r}_{0} \mid \mathbf{r}, \mathbf{r}^{\prime}, t, t^{\prime}\right) & =\left\langle w_{\alpha}\left(\mathbf{r}_{0} \mid \mathbf{r}, t\right) w_{\beta}\left(\mathbf{r}_{0} \mid \mathbf{r}^{\prime}, t^{\prime}\right)\right\rangle \\
& =\left.\frac{\delta^{2} \mathcal{Z}(\mathbf{l}, \mathbf{m})}{\delta l_{\alpha}(\mathbf{r}, t) \delta l_{\beta}\left(\mathbf{r}^{\prime}, t^{\prime}\right)}\right|_{l, m \rightarrow 0} .
\end{aligned}
$$

The perturbation theory is obtained by expanding (3.12) in $I_{\text {int }}$ which contains the fields $\mathbf{w}$ and $\mathbf{p}$. Since $I_{0}$ is a 
quadratic form in the fields, the natural objects which appear in the diagrammatic expansions are the correlators $\langle\mathbf{w w}\rangle,\langle\mathbf{w} \mathbf{p}\rangle$ and $\langle\mathbf{p p}\rangle$. The correlator $\langle\mathbf{p p}\rangle$ is zero identically in this approach [13,14,15], whereas $\langle\mathbf{w p}\rangle$ is the Green's function, or the response to an additional external noise $\mathbf{h}(\mathbf{r}, t)$ 13, 14, 15

$$
\begin{aligned}
& G_{\alpha \beta}\left(\mathbf{r}_{0} \mid \mathbf{r}, \mathbf{r}^{\prime}, t, t^{\prime}\right) \\
= & i\left\langle w_{\alpha}\left(\mathbf{r}_{0} \mid \mathbf{r}, t\right) p_{\beta}\left(\mathbf{r}^{\prime}, t^{\prime}\right)\right\rangle=\left.\frac{\delta\left\langle w_{\alpha}\left(\mathbf{r}_{0} \mid \mathbf{r}, t\right)\right\rangle}{\delta h_{\beta}\left(\mathbf{r}^{\prime}, t^{\prime}\right)}\right|_{h \rightarrow 0} .
\end{aligned}
$$

The first step in setting up the theory is the calculation of the bare Green's function. The bare Green's function $G_{\alpha \beta}^{0}$ is the response in $\mathbf{w} 3.18$ without the nonlinearity in the equation of motion. We can also introduce a Green's function $\tilde{G}_{\alpha \beta}^{0}$ which is the response of the field $\mathbf{v}$ without nonlinearity. From the equation of motion (3.7) we see that $\tilde{G}_{\alpha \beta}^{0}$ satisfies the equation

$$
\left(\frac{\partial}{\partial t}-\nu \nabla^{2}\right) \tilde{G}_{\alpha \beta}^{0}\left(\mathbf{r}, \mathbf{r}^{\prime}, t, t^{\prime}\right)=P_{\alpha \beta}\left(\mathbf{r}-\mathbf{r}^{\prime}\right) \delta\left(t-t^{\prime}\right)
$$

where the RHS stems from (3.2) with $\mathbf{a}(\mathbf{r})=\hat{e} \delta(\mathbf{r}-$ $\left.\mathbf{r}^{\prime}\right), \hat{e}$ is some unit vector. It is clear that $G_{\alpha \beta}^{0}$ can be understood as the difference

$$
G_{\alpha \beta}^{0}\left(\mathbf{r}_{0} \mid \mathbf{r}, \mathbf{r}^{\prime}, t, t^{\prime}\right)=\tilde{G}_{\alpha \beta}^{0}\left(\mathbf{r}, \mathbf{r}^{\prime}, t, t^{\prime}\right)-\tilde{G}_{\alpha \beta}^{0}\left(\mathbf{r}_{0}, \mathbf{r}^{\prime}, t, t^{\prime}\right) .
$$

From Eqs.(3.19 3.20 we conclude that for $t>t^{\prime}$ the Green's function satisfies the equation

$$
\begin{gathered}
{\left[\frac{\partial}{\partial t}-\nu\left(\nabla_{0}^{2}+\nabla^{2}\right)\right] G_{\alpha \beta}^{0}\left(\mathbf{r}_{0} \mid \mathbf{r}, \mathbf{r}^{\prime}, t, t^{\prime}\right)} \\
\quad=G_{\alpha \beta}^{0}\left(\mathbf{r}_{0} \mid \mathbf{r}, \mathbf{r}^{\prime}, 0^{+}\right) \delta\left(t-t^{\prime}\right)
\end{gathered}
$$

where

$$
G_{\alpha \beta}^{0}\left(\mathbf{r}_{0} \mid \mathbf{r}, \mathbf{r}^{\prime}, 0^{+}\right)=P_{\alpha \beta}\left(\mathbf{r}-\mathbf{r}^{\prime}\right)-P_{\alpha \beta}\left(\mathbf{r}_{0}-\mathbf{r}^{\prime}\right)
$$

The notation $t=0^{+}$means the limit $t \rightarrow 0$ from above. The functions $P_{\alpha \beta}\left(\mathbf{r}-\mathbf{r}^{\prime}\right)$ are given explicitly in Eq. (3.5). We recall that all Green's functions vanish for time $t<t^{\prime}$ due to causality. Note that the Green's function at zero time is not proportional to a delta function in $\mathbf{r}-\mathbf{r}^{\prime}$. This is a consequence of the condition of incompressibility, which means that the speed of sound is infinite, allowing information to propagate in zero time. To derive the equation for the dressed Green's function we note that the nonlinear part of the effective action has exactly the form of the Navier-Stokes nonlinearity, but in terms of $\mathbf{v}$ rather than $\mathbf{w}$. Therefore the perturbation theory that this formalism produces is identical, in terms of graphical notation and in integral representation, to the standard perturbation theory that was developed in Refs. 10,11, 13, 14. The important difference is that the objects that appear in the theory are correlators of $\mathbf{w}$ and responses of $\mathbf{w}$, and the initial condition $G_{\alpha \beta}^{0}\left(\mathbf{r}_{0} \mid \mathbf{r}\right.$, $\mathbf{r}^{\prime}, 0^{+}$) displayed in Eq. (3.23) is totally different from the initial condition in the Eulerian theory in terms of $\mathbf{u}$. In the Eulerian theory $G_{\alpha \beta}^{0}\left(0^{+}\right)$has only one projection operator $P_{\alpha \beta}\left(\mathbf{r}-\mathbf{r}^{\prime}\right)$ in contrast to $(3.22)$. This difference will have far reaching consequences as we see later. At this point however we can use the standard procedure that results in the Dyson and Wyld equations for the line renormalized correlator and Green's functions. The Dyson equation for the Green's function reads

$$
\begin{aligned}
{\left[\frac{\partial}{\partial t}\right.} & \left.-\nu\left(\nabla_{0}^{2}+\nabla^{2}\right)\right] G_{\alpha \beta}\left(\mathbf{r}_{0} \mid \mathbf{r}, \mathbf{r}^{\prime}, t\right)=G_{\alpha \beta}^{0}\left(\mathbf{r}_{0} \mid \mathbf{r}, \mathbf{r}^{\prime}, 0^{+}\right) \delta(t) \\
& +\int d \mathbf{r}_{2} G_{\alpha \delta}^{0}\left(\mathbf{r}_{0} \mid \mathbf{r}, \mathbf{r}_{2}, 0^{+}\right) \int d \mathbf{r}_{1} \int_{0}^{t} d t_{1} \\
& \times \Sigma_{\delta \gamma}\left(\mathbf{r}_{0} \mid \mathbf{r}_{2}, \mathbf{r}_{1}, t_{1}\right) G_{\gamma \beta}\left(\mathbf{r}_{0} \mid \mathbf{r}_{1}, \mathbf{r}^{\prime}, t-t_{1}\right)
\end{aligned}
$$

where $t^{\prime}$ was taken to be zero. Note that an immediate consequence of Eq. (3.23) is that at short times $\lim _{t \rightarrow 0^{+}}\left[G_{\alpha \beta}\left(\mathbf{r}_{0} \mid \mathbf{r}, \mathbf{r}^{\prime}, t\right)\right]=G_{\alpha \beta}^{0}\left(\mathbf{r}_{0} \mid \mathbf{r}, \mathbf{r}^{\prime}, 0^{+}\right)$.

The Wyld equation for the correlation function has the form

$$
\begin{aligned}
& F_{\alpha \beta}\left(\mathbf{r}_{0} \mid \mathbf{r}, \mathbf{r}^{\prime}, t\right)=\int d \mathbf{r}_{1} d \mathbf{r}_{2} \int_{0}^{\infty} d t_{1} d t_{2} G_{\alpha \gamma}\left(\mathbf{r}_{0} \mid \mathbf{r}, \mathbf{r}_{1}, t_{1}\right) \\
\times & {\left[\mathcal{D}_{\gamma \delta}\left(\mathbf{r}_{1}-\mathbf{r}_{2}, t-t_{1}+t_{2}\right)+\Phi_{\gamma \delta}\left(\mathbf{r}_{0} \mid \mathbf{r}_{1}, \mathbf{r}_{2}, t-t_{1}+t_{2}\right)\right] } \\
\times & G_{\delta \beta}\left(\mathbf{r}_{0} \mid \mathbf{r}^{\prime}, \mathbf{r}_{2}, t_{2}\right) .
\end{aligned}
$$

In equation (3.23) the mass operator $\Sigma$ is related to the "eddy viscosity" whereas in Eq. (3.24) the mass operator $\Phi$ is the renormalized "nonlinear" noise which arises due to turbulent excitations. The diagrammatic representation for $\Sigma$ and $\Phi$ is well known, and is reproduced in Fig.1.

\section{PROPERTIES OF THE DRESSED PROPAGATORS}

In this section we present those asymptotic properties of the propagators which are used in the proof of locality in Sect.4. The reader who is not interested in the details of the derivation can just pause to examine Eqs. 4.6 . 4.11) and then Eq. (4.51) before reading Sect.4.

\section{A. Rules for reading diagrams, and their physical meaning}

The objects in the diagrams of Fig.1 1 are the following: (i) the double correlator 3.16 ) of the BL-velocity differences $\mathbf{w}\left(\mathbf{r}_{0} \mid \mathbf{r}, t\right)$, which is denoted by a wavy line that carries the designation for the points $\mathbf{r}_{1}, t_{1}$ and $\mathbf{r}_{2}, t_{2}$, see Fig.11. (ii) The Green's function (3.18) which is represented half by a straight line that denotes the field $\mathbf{p}$, and half by a wavy line that stands for $\mathbf{w}$. It also carries two space-time points, see Fig.1. Note that in contrast to the standard diagrammatic approach in the terms of 
the Eulerian velocity the objects also carry a designation for the special reference point $\mathbf{r}_{0}$. (iii) The vertex $\Gamma_{\alpha \beta \gamma}$ is:

$$
\Gamma_{\alpha \beta \gamma}=\delta_{\alpha \gamma} \nabla_{\beta}+\delta_{\alpha \beta} \nabla_{\gamma}
$$

In diagrams this vertex is denoted by a bold dot that is the junction of one straight and two wavy lines. This vertex is identical to the one which appears in the standard theory in Eulerian variables 10,11], and it is local in $\mathbf{r}, t$ space. The three lines that join at the vertex carry at that point the same space-time coordinate designation. The differential operators in $\Gamma_{\alpha \beta \gamma}$ act only on the propagators that meet the vertex with wavy lines. Every diagram appearing in the expansion for $\Sigma_{\alpha \beta}\left(\mathbf{r}_{0} \mid \mathbf{r}, t \mathbf{r}^{\prime}, t^{\prime}\right)$ has an entry with a straight tail associated with $\mathbf{r}, t$ and an exit with a wavy tail associated with $\mathbf{r}^{\prime}, t^{\prime}$. Diagrams for $\Phi_{\alpha \beta}\left(\mathbf{r}_{0} \mid \mathbf{r}, t, \mathbf{r}^{\prime}, t^{\prime}\right)$ have two entries associated with straight tails carrying the designation $\mathbf{r}, t$ and $\mathbf{r}^{\prime}$ ,$t^{\prime}$ respectively, see Fig.2. Every other space-time point that appears in the inner vertices of the diagrams is integrated over. For example the one-loop diagrams for $\Sigma$ and $\Phi$ read:

$$
\begin{aligned}
& \Sigma_{\alpha \beta}\left(\mathbf{r}_{0} \mid \mathbf{r}, \mathbf{r}^{\prime}, t-t^{\prime}\right)=\left(\delta_{\alpha \epsilon} \frac{\partial}{\partial r_{\gamma}}+\delta_{\alpha \gamma} \frac{\partial}{\partial r_{\epsilon}}\right) G_{\epsilon \rho}\left(\mathbf{r}_{0} \mid \mathbf{r}, \mathbf{r}^{\prime}, t-t^{\prime}\right) \\
& \times\left(\delta_{\rho \beta} \frac{\partial}{\partial r_{\delta}^{\prime}}+\delta_{\rho \delta} \frac{\partial}{\partial r_{\beta}^{\prime}}\right) F_{\gamma \delta}\left(\mathbf{r}_{0} \mid \mathbf{r}, \mathbf{r}^{\prime}, t-t^{\prime}\right), \\
& \Phi_{\alpha \beta}\left(\mathbf{r}_{0} \mid \mathbf{r}, \mathbf{r}^{\prime}, t-t^{\prime}\right)=\frac{1}{2}\left(\delta_{\alpha \epsilon} \frac{\partial}{\partial r_{\gamma}}+\delta_{\alpha \gamma} \frac{\partial}{\partial r_{\epsilon}}\right)\left(\delta_{\beta \rho} \frac{\partial}{\partial r_{\delta}^{\prime}}\right. \\
& \left.+\delta_{\beta \delta} \frac{\partial}{\partial r_{\rho}^{\prime}}\right) F_{\gamma \delta}\left(\mathbf{r}_{0} \mid \mathbf{r}, \mathbf{r}^{\prime}, t-t^{\prime}\right) F_{\epsilon \rho}\left(\mathbf{r}_{0} \mid \mathbf{r}, r^{\prime}, t-t^{\prime}\right)
\end{aligned}
$$

Note that the RHS of (4.2) is still an operator, since the operator $\partial / \partial r_{\beta}^{\prime}$ acts on the Green's function that appears to its right in the Dyson equation (3.23). In contrast, $\Phi_{\alpha \beta}\left(\mathbf{r}_{0} \mid \mathbf{r}, t, \mathbf{r}^{\prime}, t\right)$ is a function, since the operators act only on the correlators that we see in Eq.(4.3). At the one-loop order there are no inner vertices, and therefore (4.2) and (4.3) do not have integrations. In general every diagram will have integrations over all intermediate vertices.

The physical significance of the Dyson equation is that the dressed response is determined by hydrodynamic interactions involving intermediate points. For example, the response to forcing at $\mathbf{r}^{\prime}$ has one direct, zero time contribution at $\mathbf{r}$. However, for any finite time the response to forcing at $\mathbf{r}^{\prime}$ is mediated by interactions at points $\mathbf{r}_{1}$ which via $\Sigma$ appear at point $\mathbf{r}$. In the oneloop approximation $\Sigma$ itself has a Green's function that mediates directly between $\mathbf{r}_{1}$ and $\mathbf{r}$. In higher order contributions to $\Sigma$ there are sequential contributions due to forcing at $\mathbf{r}^{\prime}$ that are mediated by responses at various $\mathbf{r}_{1}, \mathbf{r}_{2} \ldots$ until $\mathbf{r}$ is reached. $\Sigma$ represents the dressed response which is the sum of all these sequential responses in the intermediate multiple sets of points. The Green's functions that mediate intermediate points are weighted by the correlators of velocity differences between these points; if these correlators are relatively small, the contribution of the Green's function to the total response is also small.

The intuitive understanding of the Wyld equation is also straightforward. From the equation of motion (3.7), written schematically as

$$
\mathbf{w}=\frac{1}{\left[\partial / \partial t-\nu \nabla^{2}\right]}\{-[\mathbf{w} \cdot \nabla] \mathbf{w}+\tilde{\phi}\},
$$

we see that the nonlinear term $[\mathbf{w} \cdot \boldsymbol{\nabla}] \mathbf{w}$ is added to $\tilde{\phi}$ and can be understood as an "additional nonlinear noise" in the equation of motion. It is natural to expect that the double correlator of the forcing will have a nonlinear contribution of the type $\left\langle\left[\mathbf{w}\left(\mathbf{r}_{0} \mid \mathbf{r}_{1}, t_{1}\right) \boldsymbol{\nabla}_{1}\right] \mathbf{w}\left(\mathbf{r}_{0} \mid \mathbf{r}_{1}, t_{1}\right)\left[\mathbf{w}\left(\mathbf{r}_{0} \mid \mathbf{r}_{2}, t_{2}\right) \boldsymbol{\nabla}_{2}\right] \mathbf{w}\left(\mathbf{r}_{0} \mid \mathbf{r}_{2}, t_{2}\right)\right\rangle$. Indeed, the mass operator $\Phi_{\alpha \beta}\left(\mathbf{r}_{0} \mid \mathbf{r}, \mathbf{r}^{\prime}, t-t^{\prime}\right)$ can be written exactly as

$$
\begin{aligned}
& \Phi_{\alpha \beta}\left(\mathbf{r}_{0} \mid \mathbf{r}, \mathbf{r}^{\prime}, t-t^{\prime}\right) \\
= & \frac{\partial}{\partial r_{1 \gamma}} \frac{\partial}{\partial r_{2 \delta}} F_{\alpha \gamma \beta \delta}\left(\mathbf{r}_{0} \mid \mathbf{r}_{1}, \mathbf{r}_{1}, \mathbf{r}_{2}, \mathbf{r}_{2}, t_{1}, t_{1}, t_{2}, t_{2}\right),
\end{aligned}
$$

where $F_{\alpha \gamma \beta \delta}$ is the fourth order correlation of $\mathbf{w}$. Thus Eq.(3.24) can be understood as the result of squaring Eq.(4.4) and averaging, up to the dressing of the Green's functions. The content of the Wyld diagrammatics is the dressing of the bare Green's function that appears in Eq.4.4), and the representation of the fourth order $F_{\alpha \gamma \beta \delta}$ in terms of second order $F_{\alpha \beta}$. The dressing of the Green's function results from the cross terms between $[\mathbf{w} \cdot \nabla\} \mathbf{w}$ and $\tilde{\boldsymbol{\phi}}$ in (4.4). Note that the analytic form of the $\Phi_{\alpha \beta}$ in the one-loop order, Eq.(4.3) follows directly from the Gaussian decomposition of $F_{\alpha \gamma \beta \delta}$.

\section{B. Zero time properties of the renormalized propagators}

In the analysis of the asymptotic properties of the propagators it is convenient to translate the coordinate system such that $\mathbf{r}_{0}=0$. We will denote the various quantities as $G_{\alpha \beta}\left(0 \mid \mathbf{r}, \mathbf{r}^{\prime}, t\right), \Phi_{\alpha \beta}\left(0 \mid \mathbf{r}, \mathbf{r}^{\prime}, t-t^{\prime}\right)$ etc. The asymptotic properties of the bare Green's function follow from Eq.(3.22), with the transverse projector given by (3.5). Note that $G_{\alpha \beta}^{0}\left(0 \mid \mathbf{r}, \mathbf{r}^{\prime}, 0^{+}\right)$is not a symmetric function of $\mathbf{r}$ and $\mathbf{r}^{\prime}$ (also the finite time Green's function is not symmetric!). The physical meaning of $G_{\alpha \beta}^{0}\left(0 \mid \mathbf{r}, \mathbf{r}^{\prime}, 0^{+}\right)$is the zero time difference between two responses of $\mathbf{v}$ at the points $\mathbf{r}$ and 0 to forcing at $\mathbf{r}^{\prime}$. For $r^{\prime}$ much smaller that $\mathrm{r}$ the main contribution has to come from the response at the point $\mathbf{r}_{0}=0$ :

$$
G_{\alpha \beta}^{0}\left(0 \mid \mathbf{r}, \mathbf{r}^{\prime}, 0^{+}\right) \approx-P_{\alpha \beta}\left(\mathbf{r}^{\prime}\right) \sim \frac{1}{r^{\prime 3}} r^{\prime} \ll r .
$$

The next order contribution is about $1 / r^{3}$ which is much smaller. 
In studying the other limit $r \ll r^{\prime}$ we note first that for $r$ zero (i.e $\mathbf{r}=\mathbf{r}_{0}$ in arbitrary coordinates) $G_{\alpha \beta}^{0}\left(0 \mid 0, \mathbf{r}^{\prime}, 0^{+}\right)=0$ because of the exact cancellation of the two responses. For $r$ small compared to $r^{\prime}$ we can expand the difference in responses in a Taylor series to find

$$
\begin{aligned}
& G_{\alpha \beta}^{0}\left(0 \mid \mathbf{r}, \mathbf{r}^{\prime}, 0^{+}\right) \\
\approx & -r_{\gamma} \frac{\partial}{\partial r_{\gamma}^{\prime}} P_{\alpha \beta}\left(\mathbf{r}^{\prime}\right) \sim \frac{\mathbf{r} \cdot \mathbf{r}^{\prime}}{r^{\prime 5}} \quad r \ll r^{\prime} .
\end{aligned}
$$

The last form demonstrates that in this limit $G_{\alpha \beta}^{0}\left(0 \mid \mathbf{r}, \mathbf{r}^{\prime}, 0^{+}\right)$is an odd function of $\mathbf{r}$.

The most important asymptotic properties of the correlator $F_{\alpha \beta}\left(0 \mid \mathbf{r}, \mathbf{r}^{\prime}, t\right)$ for our discussion are those that appear in its $\mathrm{t}=0$ value. We can find these properties for time $t=0$ by writing $F_{\alpha \beta}\left(0 \mid \mathbf{r}, \mathbf{r}^{\prime}, t\right)$ identically as

$$
F_{\alpha \beta}\left(0, \mathbf{r}, \mathbf{r}^{\prime}, 0\right)=S_{\alpha \beta}(\mathbf{r})+S_{\alpha \beta}\left(\mathbf{r}^{\prime}\right)-S_{\alpha \beta}\left(\mathbf{r}-\mathbf{r}^{\prime}\right)
$$

where

$$
S_{\alpha \beta}(\mathbf{r}) \equiv\left\langle\left[u_{\alpha}(\mathbf{r})-u_{\alpha}(0)\right]\left[u_{\beta}(\mathbf{r})-u_{\beta}(0)\right]\right\rangle .
$$

The reason for (4.9) is that for $t=0$ the correlators of $\mathbf{v}$ coincide with the correlators of $\mathbf{u}$ as has been explained in Sec. 2. Denoting $S_{2}(r) \sim S_{\alpha \alpha}(\mathbf{r})$ we find that in the regime $r \ll r^{\prime}$ Eq. (4.8) reads

$$
F_{\alpha \beta}\left(0 \mid \mathbf{r}, \mathbf{r}^{\prime}, 0\right) \sim S_{2}(r)+r \frac{\partial S_{2}\left(r^{\prime}\right)}{\partial r^{\prime}} .
$$

Note that the function is symmetric in $\mathbf{r}, \mathbf{r}^{\prime}$, and in the opposite limit $\mathbf{r} \gg \mathbf{r}^{\prime}$ we simply have to exchange $\mathbf{r}$ and $\mathbf{r}^{\prime}$ in (4.10). For $t$ different from zero the correlator $F_{\alpha \beta}\left(0 \mid \mathbf{r}, \mathbf{r}^{\prime}, t\right)$ is always smaller (by the Cauchy-Schwarz inequality) than its value at $t=0$. This turns out to be all that we need for our discussion. The classical K41 estimate of (4.10) is

$$
F_{\alpha \beta}\left(0 \mid \mathbf{r}, \mathbf{r}^{\prime}, 0\right) \sim \bar{\epsilon}^{2 / 3}\left(r^{2 / 3}+\frac{r}{r^{1 / 3}}\right) .
$$

where $\bar{\epsilon}$ is the mean energy dissipation per unit mass per unit time.

\section{Decay of the Green's function in the short-time regime}

For the purpose of the proof of locality to be developed in Section 4 we need to control the time integrals of the Green's function. To this end we will need to estimate the characteristic decay time of $G_{\alpha \beta}\left(0 \mid \mathbf{r}, \mathbf{r}^{\prime}, t\right)$ at long times. This is done in Sec. 3.D. Here we begin by estimating the characteristic decay time $\tau_{0}\left(r, r^{\prime}\right)$ of $G_{\alpha \beta}\left(0 \mid \mathbf{r}, \mathbf{r}^{\prime}, t\right)$ at short times $t \ll \tau_{0}\left(r, r^{\prime}\right)$. We will see that the first derivative of $G_{\alpha \beta}\left(0 \mid \mathbf{r}, \mathbf{r}^{\prime}, t\right)$ vanishes at $t=0$. Therefore we define $\tau_{0}\left(\mathbf{r}, \mathbf{r}^{\prime}\right)$ in the short time regime with the help of the second derivative

$$
\left.\frac{G_{\alpha \beta}\left(0 \mid \mathbf{r}, \mathbf{r}^{\prime}, 0^{+}\right)}{\tau_{0}^{2}\left(\mathbf{r}, \mathbf{r}^{\prime}\right)} \equiv \frac{\partial^{2} G_{\alpha \beta}\left(0 \mid \mathbf{r}, \mathbf{r}^{\prime}, t\right)}{\partial t^{2}}\right|_{t=0^{+}}
$$

In principle $\tau_{0}\left(\mathbf{r}, \mathbf{r}^{\prime}\right)$ depends on the tensor indices $\alpha, \beta$. We will not be interested in numerical factors and we therefore disregard this dependence. From the point of view of the scaling theory of turbulence, typical time scales depend on the involved length scales. In K41 scaling the time scale $\tau(r)$ is proportional to $r^{2 / 3}$ :

$$
\tau(r) \sim \bar{\epsilon}^{1 / 3} r^{2 / 3}
$$

We are going to show that

$$
\tau_{0}\left(r, r^{\prime}\right) \sim \min \left\{\tau(r), \tau\left(r^{\prime}\right)\right\}=\tau\left(\min \left\{r, r^{\prime}\right\}\right) .
$$

To compute the rate of change of $G_{\alpha \beta}\left(0 \mid \mathbf{r}, \mathbf{r}^{\prime}, t\right)$ we employ the Dyson equation (3.23). For length scales $r$ and $r^{\prime}$ in the inertial range, we can neglect the viscous term in (3.23). The reason is that the viscous contribution to the time derivative (4.12) is of the order of $\nu / r^{2}$, which is much smaller than $1 / \tau(r)$. Initially we perform the evaluation of the mass operator $\Sigma$ on the RHS of (3.23) in the one-loop approximation, and then show that this evaluation is exact in the limit $t \rightarrow 0$. Substituting Eq.(4.2) into 3.23 and writing the one-loop result schematically, (disregarding tensor indices) we find for $t>0$

$$
\begin{aligned}
& \frac{\partial}{\partial t} G\left(0 \mid \mathbf{r}, \mathbf{r}^{\prime}, t\right)=\int d \mathbf{r}_{2} G^{0}\left(0 \mid \mathbf{r}, \mathbf{r}_{2}, 0^{+}\right) \int d \mathbf{r}_{1} \int_{0}^{t} d t_{1} \\
& \left.\times \frac{\partial}{\partial r_{2}} G\left(0 \mid \mathbf{r}_{2}, \mathbf{r}_{1}, t_{1}\right) \frac{\partial}{\partial r_{1}}\left[F\left(0 \mid \mathbf{r}_{2}, \mathbf{r}_{1}, t_{1}\right) G\left(0 \mid \mathbf{r}_{1}, \mathbf{r}^{\prime}, t-t_{1}\right)\right]\right) .
\end{aligned}
$$

Note that this schematic writing may be dangerous in that it obscures the possibility that some contributions might be odd under the operations $\mathbf{r}_{1} \rightarrow-\mathbf{r}_{1}$ and/or $\mathbf{r}_{2} \rightarrow-\mathbf{r}_{2}$. To see this recall that the $\partial / \partial \mathbf{r}$ operations are odd whereas the rest of the integrand has even and odd contributions. Accordingly we will take the symmetry of the integrand into account when the result depends on it.

The time arguments in $F$ and $G$ are bounded by $t$. For $t \ll \tau(r)$ we can neglect the time dependence of these propagators on the RHS of (4.15), and use their zero time values. Performing the (trivial) time integral and differentiating with respect to time we find

$$
\begin{aligned}
& \frac{\partial^{2}}{\partial t^{2}} G\left(0 \mid \mathbf{r}, \mathbf{r}^{\prime}, t\right) \approx \int d \mathbf{r}_{2} G^{0}\left(0 \mid \mathbf{r}, \mathbf{r}_{2}, 0^{+}\right) \int d \mathbf{r}_{1} \frac{\partial}{\partial r_{2}} \\
& \quad \times G^{0}\left(0 \mid \mathbf{r}_{2}, \mathbf{r}_{1}, 0^{+}\right) \frac{\partial}{\partial r_{1}}\left[F\left(0 \mid \mathbf{r}_{2}, \mathbf{r}_{1}, 0\right) G^{0}\left(0 \mid \mathbf{r}_{1}, \mathbf{r}^{\prime}, 0^{+}\right)\right] .
\end{aligned}
$$

Next we note that the two-loop order has two more time integrations that for small $t$ contribute to $\partial G\left(0 \mid \mathbf{r}, \mathbf{r}^{\prime}, t\right) / \partial t$ starting at $O\left(t^{3}\right)$, and so to Eq.(4.16) starting at $O\left(t^{2}\right)$. The n'th loop contributes starting from $O\left(t^{2 n-1}\right)$ to $\partial G\left(0 \mid \mathbf{r}, \mathbf{r}^{\prime}, t\right) / \partial t$. Thus we learn that for short times the expansion in the strength of interaction can be reformulated as an expansion in powers of the time. In the limit 
$t \rightarrow 0$ the one-loop approximation becomes exact. We can continue therefore to evaluate our quantities for short times in the one-loop order with impunity.

The analysis of the integral in (4.16) which we present next is quite cumbersome, due to the need to consider all the regimes of the relative sizes of $\mathbf{r}, \mathbf{r}^{\prime}, \mathbf{r}_{1}$ and $r_{2}$. In performing this analysis we are going to use the K41 estimate (4.11) of the correlator. We stress however that it will be shown that our conclusions are independent of the precise value of the exponents as long as they are not too far from the K41 exponents. This comment will be made quantitative later, when we understand the magnitude of the window of locality that is defined below. The result of our considerations is Eq. (4.36).

Denote the minimum and the maximum of $r, r^{\prime}$ as

$$
\lambda \equiv \min \left(r, r^{\prime}\right), \Lambda \equiv \max \left(r, r^{\prime}\right) .
$$

It will be shown that the main contribution to the integral (4.16) comes from the regime $r_{1} \sim r_{2} \sim \lambda$. We begin by showing that the regimes in which $r_{1}$ or $r_{2}$ or both are smaller than $\lambda$ contribute negligibly to the RHS of (4.16).

In the regime $r_{1}<\lambda<r_{2}$ the relevant part of the integral over $r_{1}$ in $(4.16)$ is

$$
\begin{aligned}
& \int_{0}^{\lambda} r_{1}^{2} d r_{1} G^{0}\left(0 \mid \mathbf{r}_{2}, \mathbf{r}_{1}, 0^{+}\right) \frac{\partial}{\partial r_{1}}\left[F\left(0 \mid \mathbf{r}_{2}, \mathbf{r}_{1}, 0\right) G^{0}\left(0 \mid \mathbf{r}_{1}, \mathbf{r}^{\prime}, 0^{+}\right)\right] \\
& \quad \sim \frac{1}{r^{\prime 4}} \int_{0}^{\lambda} d r_{1}\left[\frac{1}{r_{1}^{1 / 3}}+\frac{1}{r_{2}^{1 / 3}}\right] .
\end{aligned}
$$

In the estimate of the LHS integral we used the evaluations $G^{0}\left(0 \mid \mathbf{r}_{2}, \mathbf{r}_{1}, 0^{+}\right) \sim 1 / r_{1}^{3}$ [cf. Eq. (4.6)], $\partial / \partial r_{1} \sim$ $1 / r_{1}, F\left(0 \mid \mathbf{r}_{2}, \mathbf{r}_{1}, 0\right) \sim\left[r_{1}^{2 / 3}+r_{1} / r_{2}^{1 / 3}\right]$ [cf. Eq. (4.11)] and $\left.G^{0}\left(0 \mid \mathbf{r}_{1}, \mathbf{r}^{\prime},\right)^{+}\right) \sim r_{1} / r^{\prime 4}[\mathrm{cf}$. Eq.(4.7)]. We took here the next order term in $F$ because of the differentiation with respect to $r_{2}$ in (4.16). We see that in either case the main contribution to (4.18) comes from the upper limit $r_{1} \sim \lambda$.

Second we consider the regime $r_{2}<\lambda<r_{1}$. The relevant part of (4.16) now is

$$
\begin{aligned}
& \int_{0}^{\lambda} r_{2}^{2} d r_{2} G^{0}\left(0 \mid \mathbf{r}, \mathbf{r}_{2}, 0^{+}\right) \frac{\partial}{\partial r_{2}}\left[G^{0}\left(0 \mid \mathbf{r}_{2}, \mathbf{r}_{1}, 0^{+}\right) F\left(0 \mid \mathbf{r}_{2}, \mathbf{r}_{1}, 0\right)\right] \\
& \quad \sim \frac{1}{r_{1}^{4}} \int_{0}^{\lambda} \frac{d r_{2}}{r_{2}^{1 / 3}}
\end{aligned}
$$

where we used the evaluations $G^{0}\left(0 \mid \mathbf{r}, \mathbf{r}_{2}, 0^{+}\right) \sim$ $1 / r_{2}^{3}, \partial / \partial r_{2} \sim 1 / r_{2}, G^{0}\left(0 \mid \mathbf{r}_{2}, \mathbf{r}_{1}, 0^{+}\right) \sim r_{2} / r_{1}^{4}$ and $F\left(0 \mid \mathbf{r}_{2}, \mathbf{r}_{1}, 0\right) \sim r_{2}^{2 / 3}$. Again the contribution to the RHS integral is from the upper limit.

The regime $r_{1}, r_{2}<\lambda$ is more delicate. Indeed, the same kind of evaluation of the integral in 4.16) will go as follows: for $\left.r_{1} \sim r_{2}\right), G^{0}\left(0 \mid \mathbf{r}, \mathbf{r}_{2}, 0^{+}\right) \sim$ $1 / r_{2}^{3}, G^{0}\left(0 \mid \mathbf{r}_{2}, \mathbf{r}_{1}, 0^{+}\right) \sim 1 / r_{2}^{3}, F\left(0 \mid \mathbf{r}_{2}, \mathbf{r}_{1}, 0\right) \sim r_{2}^{2 / 3}$ and the integral with respect to $r_{1}$ as $r_{2}^{3}$. Using these evaluations we find

$$
\left.\frac{\partial^{2}}{\partial t^{2}} G\left(0 \mid \mathbf{r}, \mathbf{r}^{\prime}, t\right)\right|_{t=0} \sim r^{\prime-4} \int_{0}^{\lambda} d r_{2} / r_{2}^{4 / 3}, \text { wrong! }
$$

This integral indicates a divergence in the regime $r_{1} \sim$ $r_{2} \ll \lambda$. This apparent divergence is not really there; it stems from disregarding the oddness of the integral with respect to a change in sign of $\mathbf{r}_{1}$ and $\mathbf{r}_{2}$ simultaneously. The odd signature that we missed is due to the $\mathbf{r}_{2} \cdot \mathbf{r}^{\prime} / r^{\prime 5}$ [cf. Eq.(4.7)]. The rest of the integrand is even, and has been evaluated properly. Thus, looking at the next (even) order in the expansion of the last Green's function we get instead of $\mathbf{r}_{2} \cdot \mathbf{r}^{\prime} / r^{\prime 5}$ a term $\left(\mathbf{r}_{2} \cdot \mathbf{r}^{\prime}\right)^{2} / r^{\prime 7}$. Finally instead of (4.20) we get the following convergent integral

$$
\left.\frac{\partial^{2}}{\partial t^{2}} G\left(0 \mid \mathbf{r}, \mathbf{r}^{\prime}, t\right)\right|_{t=0} \sim r^{\prime-5} \int_{0}^{\lambda} d r_{2} / r_{2}^{1 / 3}, \quad \text { right ! }
$$

The other cases, namely $r_{1} \ll r_{2}<\lambda$ and $r_{2} \ll r_{1}<$ $\lambda$ can be analyzed in the same manner with the same conclusion. In summary, we learn that the integrals over $r_{1}$ and $r_{2}$ in (4.16) may take $\lambda$ as their lower limit.

Next we show that the upper limit of the integrals over $\mathbf{r}_{1}$ and $\mathbf{r}_{2}$ are bounded by $\Lambda$. In the regime $r_{1}>\Lambda>r_{2}$ the relevant part of (4.16) is similar to (4.18), and may be evaluated as

$$
\begin{aligned}
& \int_{\Lambda}^{\infty} r_{1}^{2} d r_{1} G^{0}\left(0 \mid \mathbf{r}_{2}, \mathbf{r}_{1}, 0^{+}\right) \\
\times & \frac{\partial}{\partial r_{1}}\left[F\left(0 \mid \mathbf{r}_{2}, \mathbf{r}_{1}, 0\right) G^{0}\left(0 \mid \mathbf{r}_{1}, \mathbf{r}^{\prime}, 0^{+}\right)\right] \sim \frac{r_{2}^{3}}{r^{\prime 3}} \int_{\Lambda}^{\infty} \frac{d r_{1}}{r_{1}^{13 / 3}} .
\end{aligned}
$$

To get the RHS we used the evaluations $G^{0}\left(0 \mid \mathbf{r}_{2}, \mathbf{r}_{1}, 0^{+}\right) \sim \mathbf{r}_{2} \cdot \mathbf{r}_{1} / r_{1}^{5}+\left(\mathbf{r}_{2} \cdot \mathbf{r}_{1}\right)^{2} / r_{1}^{7}, F\left(0 \mid \mathbf{r}_{2}, \mathbf{r}_{1}, 0\right) \sim$ $r_{2}^{2 / 3}+\mathbf{r}_{2} \cdot \mathbf{r}_{1} / r_{1}^{4 / 3}+\left(\mathbf{r}_{2} \cdot \mathbf{r}_{1}\right)^{2} / r_{1}^{10 / 3}$ and $G^{0}\left(0 \mid \mathbf{r}_{1}, \mathbf{r}^{\prime}, 0^{+}\right) \sim$ $1 / r^{\prime 3}$. We need to consider higher order contributions since the leading order in $F\left(0 \mid \mathbf{r}_{2}, \mathbf{r}_{1}, 0\right)$ drops in the $\partial / \partial r_{1}$ differentiation and the next order is odd. The integral in (4.22) contributes in the lower limit.

In the regime $r_{2}>\Lambda>r_{1}$ the relevant integral looks like (4.19), and is evaluated as

$$
\begin{aligned}
\int_{\Lambda}^{\infty} r_{2}^{2} d r_{2} G^{0}\left(0 \mid \mathbf{r}, \mathbf{r}_{2},\right. & \left.0^{+}\right) \frac{\partial}{\partial r_{2}}\left[G^{0}\left(0 \mid \mathbf{r}_{2}, \mathbf{r}_{1}, 0^{+}\right) F\left(0 \mid \mathbf{r}_{2}, \mathbf{r}_{1}, 0\right)\right] \\
& \sim \frac{r^{2}}{r_{1}^{11 / 3}} \int_{\Lambda}^{\infty} \frac{d r_{2}}{r_{2}^{3}} .
\end{aligned}
$$

In this case we evaluated $G^{0}\left(0 \mid \mathbf{r}, \mathbf{r}_{2}, 0^{+}\right) \sim \mathbf{r} \cdot \mathbf{r}_{2} / r_{2}^{5}+$ $\left(\mathbf{r} \cdot \mathbf{r}_{2}\right)^{2} / r_{2}^{7}, G^{0}\left(0 \mid \mathbf{r}_{2}, \mathbf{r}_{1}, 0^{+}\right) \sim 1 / r_{1}^{3}, F\left(0 \mid \mathbf{r}_{2}, \mathbf{r}_{1}, 0\right) \sim$ $r_{1}^{2 / 3}+\mathbf{r}_{1} \cdot \mathbf{r}_{2} / r_{1}^{4 / 3}+\left(\mathbf{r}_{1} \cdot \mathbf{r}_{2}\right)^{2} / r_{1}^{10 / 3}$. We need the higher order terms for the same reasons as discussed above. This integral also contributes at $\Lambda$.

Finally we need to consider the regime $r_{1} \sim r_{2}>\Lambda$. The leading order evaluation is again zero by symmetry. Taking into account the next order contribution we evaluate

$$
\left.\frac{\partial^{2}}{\partial t^{2}} G\left(0 \mid \mathbf{r}, \mathbf{r}^{\prime}, t\right)\right|_{t=0^{+}} \sim \frac{r^{2}}{r^{\prime 3}} \int_{\Lambda}^{\infty} \frac{d r_{2}}{r_{2}^{13 / 3}}
$$


In summary, the contribution to the integrals over $r_{1}$ and $r_{2}$ in (4.16) from regions much smaller than $\lambda$ and much larger than $\Lambda$ are negligible. This is the first example of the property that we refer to in this paper as "locality". This property allows us to estimate $(4.16)$ as the definite integral

$$
\begin{aligned}
& \left.\frac{\partial^{2}}{\partial t^{2}} G\left(0 \mid \mathbf{r}, \mathbf{r}^{\prime}, t\right)\right|_{t=0^{+}} \sim \int_{\lambda}^{\Lambda} d r_{2} r_{2}^{2} G^{0}\left(0 \mid \mathbf{r}, \mathbf{r}_{2}, 0^{+}\right) \int_{\lambda}^{\Lambda} d r_{1} r_{1}^{2} \frac{\partial}{\partial r_{2}} \\
& \times G^{0}\left(0 \mid \mathbf{r}_{2}, \mathbf{r}_{1}, 0^{+}\right) \frac{\partial}{\partial r_{1}}\left[F\left(0 \mid \mathbf{r}_{2}, \mathbf{r}_{1}, 0\right) G^{0}\left(0 \mid \mathbf{r}_{1}, \mathbf{r}^{\prime}, 0^{+}\right)\right] .
\end{aligned}
$$

The integral (4.25) offers us an easy evaluation of the characteristic time $\tau\left(r, r^{\prime}\right)$ when $\mathrm{r}$ and r' are of the same order, i.e. when $\lambda=O(\Lambda)$. In this case we simply power count and find that

$$
\left.\frac{\partial^{2}}{\partial t^{2}} G\left(0 \mid \mathbf{r}, \mathbf{r}^{\prime}, t\right)\right|_{t=0^{+}} \sim \frac{1}{r^{13 / 3}} .
$$

Comparing this result with Eq.4.12 shows that

$$
\tau_{0}(r, r) \sim \bar{\epsilon}^{-1 / 3} r^{2 / 3},
$$

which is identical to the Kolmogorov time $\tau(r)$ given by (4.13). We note that this finding is far from trivial; it requires the proof of locality. Once locality is shown, the analytical result must agree with dimensional analysis as we indeed find. It is important to understand however that Eq. (4.25) offers more than can be achieved by dimensional analysis even with locality. For example we can discuss now $\tau_{0}\left(r, r^{\prime}\right)$ when $r \gg r^{\prime}$ and $r \ll r^{\prime}$. Since the ratio $r / r^{\prime}$ is dimensionless, dimensional analysis cannot be employed to estimate the characteristic time $\tau\left(r, r^{\prime}\right)$ in such cases.

The first case to be discussed is $r \ll r^{\prime}$. We use Eq.4.25 with $\lambda=r$ and $\Lambda=r^{\prime}$. The first and last Green's functions in (4.25) are evaluated as $G^{0}\left(0 \mid \mathbf{r}, \mathbf{r}_{2}, 0^{+}\right) \sim r / r_{2}^{4}$ and $G^{0}\left(0 \mid \mathbf{r}_{1}, \mathbf{r}^{\prime}, 0^{+}\right) \sim r_{1} / r^{\prime 4}$. This leads to the integral

$$
\begin{aligned}
& \left.\frac{\partial^{2}}{\partial t^{2}} G\left(0 \mid \mathbf{r}, \mathbf{r}^{\prime}, t\right)\right|_{t=0^{+}} \\
\sim & \frac{r}{r^{\prime 4}} \int_{r}^{r^{\prime}} \frac{d r_{2}}{r_{2}^{2}} \int_{r}^{r^{\prime}} d r_{1} r_{1}^{2} \frac{\partial}{\partial r_{2}} G^{0}\left(0 \mid \mathbf{r}_{2}, \mathbf{r}_{1}, 0^{+}\right) F\left(0 \mid \mathbf{r}_{2}, \mathbf{r}_{1}, 0\right)
\end{aligned}
$$

We break now the integration over $r_{1}$ into the two domains $\left(r, r_{2}\right)$ and $\left(r_{2}, r^{\prime}\right)$. In the first domain the $r_{1}$ integrand is evaluated as $1 / r_{2}^{4 / 3}$, and as $r_{2}^{2 / 3} / r_{1}^{2}$ in the second domain. In both domains therefore the main contribution comes for the regime $r_{1} \sim r_{2}$. Thus the $r_{1}$ integral is evaluated as $1 / r_{2}^{1 / 3}$. With this we can see that the $r_{2}$ integral in (4.28) contributes mainly at $r_{2} \sim r$, and can be evaluated as $1 / r^{4 / 3}$. Finally

$$
\left.\frac{\partial^{2}}{\partial t^{2}} G\left(0 \mid \mathbf{r}, \mathbf{r}^{\prime}, t\right)\right|_{t=0^{+}} \sim \frac{1}{r^{\prime 4} r^{1 / 3}}, \quad r \ll r^{\prime} .
$$

Remembering that in this regime $G^{0}\left(0 \mid \mathbf{r}, \mathbf{r}^{\prime}, 0^{+}\right) \sim r / r^{4}$ we conclude with 4.12 ) that

$$
\tau\left(r, r^{\prime}\right) \sim(\bar{\epsilon})^{-1 / 3} r^{2 / 3} \sim \tau(r), \quad r \ll r^{\prime} .
$$

In the opposite limiting case, i.e. $r \gg r^{\prime}$, Eq. (4.25) is employed with $\lambda=r^{\prime}$ and $\Lambda=r$. After the evaluation of the first and last Green's functions the integral in 4.25) reads:

$$
\begin{aligned}
& \left.\frac{\partial^{2}}{\partial t^{2}} G\left(0 \mid \mathbf{r}, \mathbf{r}^{\prime}, t\right)\right|_{t=0^{+}} \sim r^{\prime-3} \int_{r^{\prime}}^{r} \frac{d r_{2}}{r_{2}} \\
\times & \int_{r^{\prime}}^{r} d r_{1} r_{1}^{2} \frac{\partial}{\partial r_{2}} G^{0}\left(0 \mid \mathbf{r}_{2}, \mathbf{r}_{1}, 0^{+}\right) \frac{\partial}{\partial r_{1}} F\left(0 \mid \mathbf{r}_{2}, \mathbf{r}_{1}, 0\right)
\end{aligned}
$$

We split again the $r_{1}$ integral into two domains of integration $\left(r^{\prime}, r_{2}\right)$ and $\left(r_{2}, r\right)$. In the first domain we evaluate $G^{0}\left(0 \mid \mathbf{r}_{2}, \mathbf{r}_{1}, 0^{+}\right) \sim 1 / r_{1}^{3}$, and $\partial^{2}\left[F\left(0 \mid \mathbf{r}_{2}, \mathbf{r}_{1}, 0\right)\right] / \partial r_{1} \partial r_{2} \sim$ $1 / r_{2}^{4 / 3}$. Substituting in the $r_{1}$ integral we evaluate it as $\ln \left(r_{2} / r^{\prime}\right) / r_{2}^{4 / 3}$. Evaluating then the $r_{2}$ integral we find that it contributes at $r_{2} \sim r^{\prime}$. We end up with

$$
\left.\frac{\partial^{2}}{\partial t^{2}} G\left(0 \mid \mathbf{r}, \mathbf{r}^{\prime}, t\right)\right|_{t=0^{+}} \sim \frac{1}{r^{13 / 3}} .
$$

In the second domain with $r_{1}>r_{2}$ we have to take into account two terms in the evaluation of $G^{0}\left(0 \mid \mathbf{r}_{2}, \mathbf{r}_{1}, 0^{+}\right)$, since the contribution of the first one will vanish by symmetry $G^{0}\left(0 \mid \mathbf{r}_{2}, \mathbf{r}_{1}, 0^{+}\right) \sim \mathbf{r}_{2} \cdot \mathbf{r}_{1} / r_{1}^{5}+\left(\mathbf{r}_{2} \cdot \mathbf{r}_{1}\right)^{2} / r_{1}^{7}$. Evaluating $\partial F\left(0 \mid \mathbf{r}_{2}, \mathbf{r}_{1}, 0\right) / \partial r_{1} \sim r_{2} / r_{1}^{4 / 3}$ we end up with the evaluation of the $r_{1}$ integrand as $r_{2}^{2} / r_{1}^{13 / 3}$. The $r_{1}$ integral contributes at $r_{1} \sim r_{2}$ and is evaluated as $1 / r_{2}^{7 / 3}$. The $r_{2}$ integral contributes at $r^{\prime}$, and can be evaluated as $1 / r^{\prime 4 / 3}$. This means that the two domains of integration lead to the same evaluation (4.32) which is therefore the final evaluation in this regime of $r \gg r^{\prime}$. Remembering that here $G^{0}\left(0 \mid \mathbf{r}, \mathbf{r}^{\prime}, 0^{+}\right) \sim 1 / r^{\prime 3}$ we end up, using 4.12) with

$$
\tau_{0}\left(r, r^{\prime}\right) \sim(\bar{\epsilon})^{-1 / 3} r^{2 / 3} \sim \tau\left(r^{\prime}\right), r^{\prime} \ll r .
$$

The conclusion from Eqs. $(4.30)$ and $(4.33)$ is that in the short time regime the typical decay time of the Green's function $\tau\left(r, r^{\prime}\right)$ is always of the order of the smaller turn over times, as written in (4.14). This conclusion is intuitively quite reasonable.

It is very important to notice that we have proven here locality in a stronger sense than the one invoked in (4.25). It was shown that both the $r_{1}$ and the $r_{2}$ integrals take their main contribution from the regime $r_{1} \sim r_{2} \sim \lambda=$ $\min \left(r, r^{\prime}\right)$. Although we used the Kolmogorov scaling for the evaluation of the integral, the result does not depend on that. We have a wide window of locality. We can change the scaling exponents of $F\left(0 \mid \mathbf{r}_{2}, \mathbf{r}_{1}, 0\right)$ and/or of $\tau(r)$ by a small number and still all our integrals will continue to be dominated by the same domains $r_{1} \sim$ $r_{2} \sim \lambda$.

In fact, we can derive the result (4.14) for the typical time scale without invoking any numerical value for the scaling exponents. Assume that all the integrals in 4.25) are local in the sense that the largest contribution comes 
from the region of integration $r_{1} \sim r_{2} \sim \lambda=\min \left(r, r^{\prime}\right)$. Under this assumption we can evaluate $G^{0}\left(0 \mid \mathbf{r}_{1}, \mathbf{r}_{2}, 0^{+}\right) \sim$ $1 / \lambda^{3}, F\left(0 \mid \mathbf{r}_{2}, \mathbf{r}_{1}, 0\right) \sim S_{2}(\lambda)$ and $\partial / \partial r_{1} \sim \partial / \partial r_{2} \sim \lambda^{-1}$. Eq. (4.25) takes the form

$$
\left.\frac{\partial^{2}}{\partial t^{2}} G\left(0 \mid \mathbf{r}, \mathbf{r}^{\prime}, t\right)\right|_{t=0^{+}} \sim \lambda G^{0}\left(0 \mid r, \lambda, 0^{+}\right) S_{2}(\lambda) G^{0}\left(0 \mid \lambda, r^{\prime}, 0^{+}\right) \text {. }
$$

Using Eq. 4.12 we find

$$
\tau_{0}^{2}\left(r, r^{\prime}\right) \sim \frac{G\left(0 \mid \mathbf{r}, \mathbf{r}^{\prime}, 0^{+}\right)}{\lambda G^{0}\left(0 \mid r, \lambda, 0^{+}\right) S_{2}(\lambda) G^{0}\left(0 \mid \lambda, r^{\prime}, 0^{+}\right)} .
$$

Consider now the limiting case $r \ll r^{\prime}$. Now $\lambda=r$ and $\Lambda=r^{\prime}$, and we can estimate $G\left(0 \mid \mathbf{r}, \mathbf{r}^{\prime}, 0^{+}\right) \sim$ $\lambda / \Lambda^{4}, G^{0}\left(0 \mid r, \lambda, 0^{+}\right) \sim 1 / \lambda^{3}$ and $G^{0}\left(0 \mid \lambda, r^{\prime}, 0^{+}\right) \sim \lambda / \Lambda^{4}$. Finally we get again (4.14) but with a more general expression for $\tau(\lambda)$ :

$$
\begin{aligned}
\tau_{0}\left(r, r^{\prime}\right) & =\tau(\lambda), \\
\tau(\lambda) & \approx \frac{\lambda}{\sqrt{S_{2}(\lambda)}} .
\end{aligned}
$$

In the opposite case in which $\Lambda=r \gg r^{\prime}=\lambda$ all the Green's functions are evaluated as $1 / \lambda^{3}$ and we regain (4.36). Thus (4.36) is the final result for the characteristic time scale. This time scale is recognized as the typical turnover time of eddies of size $\lambda$, having rms velocity of $\sqrt{S_{2}(\lambda)}$. Clearly for K41 scaling we recapture the scaling law $\tau(\lambda) \sim \lambda^{2 / 3}$, but in general our result is that the decay time of the Green's function $G\left(0 \mid \mathbf{r}, \mathbf{r}^{\prime}, t\right)$ is the turn over time of eddies of scale $\lambda=\min \left(r, r^{\prime}\right)$, independently of the exponent. Eq.(4.37) is one of the scaling relations between exponents of static and dynamic quantities that are used later to find the scaling solution of our theory.

Note that the typical time scale $\tau(r)$ becomes the only relevant time scale of $G\left(0 \mid \mathbf{r}, \mathbf{r}^{\prime}, t\right)$ when $r$ is about $r^{\prime}$. In fact, when $r \sim r^{\prime}$ this is also the typical time scale of the correlator $F\left(0 \mid \mathbf{r}, \mathbf{r}^{\prime}, t\right)$, of the mass operator $\Sigma\left(0 \mid \mathbf{r}, \mathbf{r}^{\prime}, t\right)$, etc. The reason is that the problem contains no dimensional parameters, and therefore all the objects of the theory with the same spatial scale $\mathrm{r}$ must have the same characteristic time $\tau(r)$. This is not an assumption, but a consequence of the structure of the theory and the property of locality that is proven in full in Sec.4.

\section{Characteristic decay time of the Green's function}

In the previous section we evaluated the characteristic decay time $\tau_{0}\left(r, r^{\prime}\right)$ of $G\left(0 \mid \mathbf{r}, \mathbf{r}^{\prime}, t\right)$ in the short time regime $t \ll \tau_{0}\left(r, r^{\prime}\right)$. For the proof of locality we need to evaluate time integrals over $G\left(0 \mid \mathbf{r}, \mathbf{r}^{\prime}, t\right)$, and for this purpose we need to estimate the global decay time $\tau\left(r, r^{\prime}\right)$ defined as

$$
\int_{0}^{\infty} d t G\left(0 \mid \mathbf{r}, \mathbf{r}^{\prime}, t\right)=\tau\left(r, r^{\prime}\right) G^{0}\left(0 \mid \mathbf{r}, \mathbf{r}^{\prime}, 0^{+}\right)
$$

Of course this definition makes sense only if the integral converges; we prove this fact next. For $r$ and $r^{\prime}$ of different orders we have no a priori reason to expect that $\tau\left(r, r^{\prime}\right)$ is of the order of $\tau_{0}\left(r, r^{\prime}\right)$.

To prove the convergence of the integral in (4.38) we integrate Eq.(3.23) in time between $t=-\epsilon$ and infinity. For $r, r^{\prime}$ in the inertial interval we neglect the viscous term for the same reasons as discussed in Section 3C. We find

$$
\begin{aligned}
& \int_{-\epsilon}^{\infty} \frac{\partial}{\partial t} G_{\alpha \beta}\left(0 \mid \mathbf{r}, \mathbf{r}^{\prime}, t\right) d t=G_{\alpha \beta}^{0}\left(0 \mid \mathbf{r}, \mathbf{r}^{\prime}, 0^{+}\right) \\
+ & \int_{-\epsilon}^{\infty} d t \int d \mathbf{r}_{2} G_{\alpha \delta}^{0}\left(0 \mid \mathbf{r}, \mathbf{r}_{2}, 0^{+}\right) \int d \mathbf{r}_{1} \int_{0}^{t} d t_{1} \\
\times & \Sigma_{\delta \gamma}\left(0 \mid \mathbf{r}_{2}, \mathbf{r}_{1}, t_{1}\right) G_{\gamma \beta}\left(0 \mid \mathbf{r}_{1}, \mathbf{r}^{\prime}, t-t_{1}\right) .
\end{aligned}
$$

The Green's function vanishes for $t \rightarrow \infty$, and it is identically zero for all negative times. Accordingly, the LHS is zero, and after changing the order of integration over time on the RHS we arrive at

$$
\begin{aligned}
& G_{\alpha \beta}^{0}\left(0 \mid \mathbf{r}, \mathbf{r}^{\prime}, 0^{+}\right)=-\int d \mathbf{r}_{2} G_{\alpha \delta}^{0}\left(0 \mid \mathbf{r}, \mathbf{r}_{2}, 0^{+}\right) \int d \mathbf{r}_{1} \int_{0}^{\infty} d t_{1} \\
& \times \Sigma_{\delta \gamma}\left(0 \mid \mathbf{r}_{2}, \mathbf{r}_{1}, t_{1}\right) \int_{0}^{\infty} d t G_{\alpha \beta}\left(0 \mid \mathbf{r}_{1}, \mathbf{r}^{\prime}, t\right) .
\end{aligned}
$$

Since the LHS is finite, both time integrals on the RHS converge. Thus the definition (4.38) is meaningful, and we proceed to estimate $\tau\left(r, r^{\prime}\right)$.

To evaluate $\tau\left(r, r^{\prime}\right)$ we forget the tensor indices, and then begin the analysis by assuming that the main contribution to (4.40) comes from the regime $r_{1} \sim r_{2}$. Evaluating $\int d \mathbf{r}_{2} \sim r_{1}^{3}$, and making use of (4.38) we rewrite (4.40) as

$$
\begin{array}{r}
G^{0}\left(0 \mid \mathbf{r}, \mathbf{r}^{\prime}, 0^{+}\right)=\int r_{1}^{5} d r_{1} G^{0}\left(0 \mid \mathbf{r}, \mathbf{r}_{1}, 0^{+}\right) \int_{0}^{\infty} d t_{1} \\
\times \Sigma\left(0 \mid \mathbf{r}_{1}, \mathbf{r}_{1}, t_{1}\right) \tau\left(r_{1}, r^{\prime}\right) G^{0}\left(0 \mid \mathbf{r}_{1}, \mathbf{r}^{\prime}, 0^{+}\right)
\end{array}
$$

Since we proved that both time integrals in (4.40) are finite, the integral $\int_{0}^{\infty} d t_{1} \Sigma\left(0 \mid \mathbf{r}_{1}, \mathbf{r}_{1}, t_{1}\right)$ can be evaluated as $\tau\left(r_{1}\right) \Sigma\left(0 \mid \mathbf{r}_{1}, \mathbf{r}_{1}, t_{1}=0\right)$. The time scale $\tau\left(r_{1}\right)$ is the same as the one used in the context of the Green's function, because there is only one spatial scale available here. The mass operator $\Sigma\left(0 \mid \mathbf{r}_{1}, \mathbf{r}_{1}, t_{1}=0\right)$ can be evaluated in the one-loop approximation (4.2) as $S_{2}(r) / r^{5}$. Using the scaling relation (4.37) this is equivalent to

$$
\Sigma\left(0 \mid \mathbf{r}_{1}, \mathbf{r}_{1}, t_{1}=0\right) \sim \frac{1}{r_{1}^{3} \tau^{2}\left(r_{1}\right)}
$$

Thus we can write

$$
\begin{aligned}
& G^{0}\left(0 \mid \mathbf{r}, \mathbf{r}^{\prime}, 0^{+}\right) \\
\sim & \int r_{1}^{2} d r_{1} G^{0}\left(0 \mid \mathbf{r}, \mathbf{r}_{1}, 0^{+}\right) \frac{\tau\left(r_{1}, r^{\prime}\right)}{\tau\left(r_{1}\right)} G^{0}\left(0 \mid \mathbf{r}_{1}, \mathbf{r}^{\prime}, 0^{+}\right) .
\end{aligned}
$$

We are going to explore the implications of (4.43) on the allowed behavior of the ratio $\tau\left(r_{1}, r^{\prime}\right) / \tau\left(r_{1}\right)$. As discussed 
above, for $r_{1} \sim r^{\prime}$ this ratio should be of $O(1)$. For $r_{1} \gg r^{\prime}$ but both $\mathrm{r}$ and $r^{\prime}$ in the inertial interval we can expect a scaling behavior of the ratio

$$
\frac{\tau\left(r_{1}, r^{\prime}\right)}{\tau\left(r_{1}\right)} \sim\left[\frac{r^{\prime}}{r_{1}}\right]^{\alpha}, \quad r_{1} \gg r^{\prime}
$$

with some scaling exponent $\alpha$. Similarly, for $r_{1} \ll r^{\prime}$, we expect that

$$
\frac{\tau\left(r_{1}, r^{\prime}\right)}{\tau\left(r_{1}\right)} \sim\left[\frac{r^{\prime}}{r_{1}}\right]^{\beta}, \quad r_{1} \ll r^{\prime} .
$$

Consider now (4.43) in the domain $(\Lambda, \infty)$. The evaluation of the integrand is straightforward, and we write

$$
G^{0}\left(0 \mid \mathbf{r}, \mathbf{r}^{\prime}, 0^{+}\right) \sim \int_{\Lambda}^{\infty} \frac{d r_{1}}{r_{1}^{2}}\left[\frac{r^{\prime}}{r_{1}}\right]^{\alpha}
$$

Convergence in the upper limit, which is guaranteed by the fact that the LHS is finite, requires $\alpha>-1$. In the domain $(0, \lambda)$ we evaluate

$$
G^{0}\left(0 \mid \mathbf{r}, \mathbf{r}^{\prime}, 0^{+}\right) \sim \int_{0}^{\lambda} d r_{1}\left[\frac{r^{\prime}}{r_{1}}\right]^{\beta} .
$$

Convergence in the lower limit requires $\beta<1$. With these restrictions $(\alpha>-1$ and $\beta<1$ ) the main contribution to the integral (4.43) comes from the domain $(\lambda, \Lambda)$. For $r \gg r^{\prime}$ we evaluate the LHS and RHS of (4.43) as

$$
\frac{1}{r^{\prime 3}} \sim \frac{1}{r^{\prime 3}} \int_{r^{\prime}}^{r} \frac{d r_{1}}{r_{1}}\left[\frac{r^{\prime}}{r_{1}}\right]^{\alpha} .
$$

In order to satisfy this equation the integral must contribute in the lower limit. Thus $\alpha$ must satisfy $\alpha>0$. This is a stronger constraint than the one required by convergence.

For $r \ll r^{\prime}$ we evaluate the LHS and RHS of (4.43) as

$$
\frac{r}{r^{\prime 4}} \sim \frac{r}{r^{\prime 4}} \int_{r}^{r^{\prime}} \frac{d r_{1}}{r_{1}}\left[\frac{r^{\prime}}{r_{1}}\right]^{\beta}
$$

This integral must contribute in the upper limit in order to satisfy the equation. This requires $\beta<0$. Combining these results with (4.44) and (4.45) we reach the final conclusion that

$$
\tau\left(r, r^{\prime}\right) / \tau(r)<\mathrm{const}
$$

with const $=\mathrm{O}(1)$ independently of the relation between $r$ and $r^{\prime}$. In terms of our goal which was the estimate of the integral over the Green's function, we can write our final conclusion in the form

$$
\int_{0}^{\infty} d t G_{\gamma \beta}\left(0 \mid \mathbf{r}, \mathbf{r}^{\prime}, t\right)<\tau(r) G_{\alpha \beta}^{0}\left(0 \mid \mathbf{r}, \mathbf{r}^{\prime}, 0^{+}\right) ; \text {always! }
$$

The meaning of this is deep: this equation says that the characteristic time of decay of the Green's function $G_{\gamma \beta}\left(0 \mid \mathbf{r}, \mathbf{r}^{\prime}, t\right)$ is always bounded by the characteristic time of the first coordinate $(\mathbf{r})$ which is the coordinate designating the difference between the two responses at $\mathbf{r}$ and 0 . In the coordinate system with $\mathbf{r}_{0} \neq 0$ the decay of the Green's function is bounded by the time scale of $\left|\mathbf{r}-\mathbf{r}_{0}\right|$.

We remember that up to now we considered the local contribution $r_{1} \sim r_{2}$. There are good reasons to expect that this is the largest contribution. However, if it turns out that this is not the case, then Eq.(4.40) implies that the estimate of the characteristic time scale $\tau\left(r, r^{\prime}\right)$ is even smaller than the bound $(4.50)$. Therefore the bound (4.51) can be used safely.

\section{PROOF OF LOCALITY}

In this section we prove that all the diagrams for the Green's function and correlators are dominated by interactions within a shell of a ball in physical space whose radius $r$ will be determined later. The logic of the arguments is as follows. It is easy to evaluate an arbitrary diagram given that the coordinates $\mathbf{r}_{j}$ of all vertices lie within a ball of radius $r$. The aim is to show that any contributions in which any of these vertices lies outside the ball of radius $r$ is smaller that this "local" contributions. We will begin with the case in which only one vertex lies outside, and analyze the different geometries in which this may occur. We than consider the case of a group of vertices which lie close to each other but outside the ball, and finally the case where there are two separated groups of vertices lying outside the ball. This can be than generalized to any number of groups. The fact that all our integrals converge in both the upper and the lower limits serves as a proof that there are no perturbative mechanisms to renormalize the K41 exponents. This is the main point of this paper.

\section{A. The analysis of outer fragments of diagrams}

We begin by proving that if the coordinate of one vertex, say $\mathbf{r}_{1}$, in an arbitrary diagram is removed far away from all the rest of the vertices, the effect is to reduce the evaluation of the diagram as an inverse power of $r_{1}$. Later we will use this result in our analysis of arbitrarily complicated diagrams. To facilitate the examination of the diagrams we will draw them in $\mathbf{r}, t$ space, such that vertices at a larger value of $\mathrm{r}$ will be positioned above vertices at smaller values of $r$, and larger times will be to the left of smaller times. Consider now a fragment of an arbitrary diagram in which one vertex whose coordinate $\mathbf{r}_{1}$ is lying above all the other vertices in the diagram. In Fig. 3 we show the three different possibilities of such a vertex at $\mathbf{r}_{1}$ which is connected to another vertex at 
smaller $\mathbf{r}$ via a Green's function. The relative positions in space and time of the four adjacent vertices are clear from the pictures. We always take here $r_{1}>r$ and our aim is to show that the major contribution to the integral over $\mathbf{r}_{1}$ comes from the region $r_{1} \approx r$. Note that due to causality the vertex at $\mathbf{r}_{1}$ must be to the right of the vertex at $\mathbf{r}$, and any other Green's function that starts at $\mathbf{r}_{1}$ has to point to the right. The wavy lines of the correlators can go either right or left, and we show only one representative example for each diagram that includes correlators. Since we are going to estimate the correlators by their $t=0$ value, the time coordinates of the correlators do not matter. The three examples of Fig, 3 are denoted as $I_{a}, I_{b}$ and $I_{c}$. The analytic expressions for these three examples are shown below. For simplicity we do not display tensor indices:

$$
\begin{aligned}
I_{a} & \sim \int_{r}^{\infty} d \mathbf{r}_{1} \int_{t}^{\infty} d t_{1} \frac{\partial}{\partial r} G\left(0 \mid \mathbf{r}, \mathbf{r}_{1}, t_{1}-t\right) \\
& \times \frac{\partial}{\partial r_{1}}\left[F\left(0 \mid \mathbf{r}_{1}, \mathbf{r}_{2}, t_{1}-t_{2}\right) F\left(0 \mid \mathbf{r}_{1}, \mathbf{r}_{3}, t_{1}-t_{3}\right)\right]
\end{aligned}
$$

We are going to bound $I_{a}$ from above. Accordingly, we take the largest possible value of the correlators, which is the $t=0$ value. After that we can bound the integral over $t_{1}$ by using (4.51). We use the symbols $\sim$ and $\propto$ in our evaluations, but we remember that we are actually estimating upper bounds; the quantities are smaller than what is displayed:

$$
I_{a} \propto \tau(r) \int_{r}^{\infty} \frac{d r_{1}}{r_{1}^{2}} \frac{\partial}{\partial r_{1}}\left[F\left(0 \mid \mathbf{r}_{1}, \mathbf{r}_{2}, 0\right) F\left(0 \mid \mathbf{r}_{1}, \mathbf{r}_{3}, 0\right)\right]
$$

We now evaluate

$$
\frac{\partial}{\partial r_{1}}\left[F\left(0 \mid \mathbf{r}_{1}, \mathbf{r}_{2}, 0\right) F\left(0 \mid \mathbf{r}_{1}, \mathbf{r}_{3}, 0\right)\right] \approx \bar{\epsilon}^{4 / 3} r_{i}^{2 / 3} r_{j} / r_{1}^{4 / 3}
$$

where $r_{j}$ is $\max \left\{r_{2}, r_{3}\right\}$, and $r_{i}=\min \left\{r_{2}, r_{3}\right\}$. Obviously, the integrals in $(5.2)$ contributes at $r_{1} \approx r$.

The integral $I_{b}$ is

$$
\begin{aligned}
I_{b} & \sim \int d \mathbf{r}_{1} \int_{t}^{t_{2}} d t_{1} \frac{\partial}{\partial r} G\left(0 \mid \mathbf{r}, \mathbf{r}_{1}, t_{1}-t\right) \\
& \times \frac{\partial}{\partial r_{1}}\left[G\left(0 \mid \mathbf{r}_{1}, \mathbf{r}_{2}, t_{1}-t_{2}\right) F\left(0 \mid \mathbf{r}_{1}, \mathbf{r}_{3}, t_{1}-t_{3}\right)\right]
\end{aligned}
$$

In evaluating this integral we need to remember that in the generic diagrams the coordinate $t_{2}$ is integrated upon. The characteristic decay time of the Green's function $G\left(0 \mid \mathbf{r}_{1} \mathbf{r}_{2}, t_{1}-t_{2}\right)$ is bounded by $\tau\left(r_{1}\right)$. However because this Green's function is connected to other propagators via vertex 2 , the time restriction is at most $\tau\left(r_{2}\right)$ and not $\tau\left(r_{1}\right)$. Thus we write

$$
I_{b} \propto \tau(r) \int_{r}^{\infty} \frac{d r_{1}}{r_{1}^{2}} \frac{\partial}{\partial r_{1}}\left[G\left(0 \mid \mathbf{r}_{1}, \mathbf{r}_{2}, 0\right) F\left(0 \mid \mathbf{r}_{1}, \mathbf{r}_{3}, 0\right)\right] .
$$

We can evaluate now

$$
\frac{\partial}{\partial r_{1}}\left[G\left(0 \mid \mathbf{r}_{1}, \mathbf{r}_{2}, 0\right) F\left(0 \mid \mathbf{r}_{1}, \mathbf{r}_{3}, 0\right)\right] \approx \bar{\epsilon}^{2 / 3} \frac{\hat{\mathbf{r}}_{1} \cdot \mathbf{r}_{2}}{r_{3}^{3} r_{1}^{2 / 3}} .
$$

We see that the integral (5.5) is local, contributing mostly at $r_{1} \sim r$.

Next we consider the integral $I_{c}$ :

$$
\begin{aligned}
I_{c} & \sim \int d \mathbf{r}_{1} \int_{t}^{t_{i}} d t_{1} \frac{\partial}{\partial r} G\left(0 \mid \mathbf{r}, \mathbf{r}_{1}, t_{1}-t\right) \\
& \times \frac{\partial}{\partial r_{1}}\left[G\left(0 \mid \mathbf{r}_{1}, \mathbf{r}_{2}, t_{1}-t_{2}\right) G\left(0 \mid \mathbf{r}_{1}, \mathbf{r}_{3}, t_{1}-t_{3}\right)\right]
\end{aligned}
$$

where $t_{i}=\min \left[t_{1}, t_{2}\right]$. In this case the integral $\int G\left[0 \mid \mathbf{r}_{1}, \mathbf{r}_{3},\left(t_{1}-t_{3}\right)\right] d t_{1}$ does contribute a factor bounded by $\tau\left(r_{1}\right) G\left(0 \mid \mathbf{r}_{1}, \mathbf{r}_{3}, 0^{+}\right)$. We thus need to take into account the factor $\tau\left(r_{1}\right)$ in the integrand and write

$$
I_{c} \propto \tau(r) \int_{r}^{\infty} \frac{d r_{1}}{r_{1}^{2}} \frac{\partial}{\partial r_{1}}\left[\tau\left(r_{1}\right) G\left(0 \mid \mathbf{r}_{1}, \mathbf{r}_{2}, 0\right) G\left(0 \mid \mathbf{r}_{1}, \mathbf{r}_{3}, 0\right)\right]
$$

In the present case we use (4.6) and 4.7) to write

$$
\frac{\partial}{\partial r_{1}}\left[G\left(0 \mid \mathbf{r}_{1}, \mathbf{r}_{2}, 0\right) G\left(0 \mid \mathbf{r}_{1}, \mathbf{r}_{3}, 0\right)\right] \sim \frac{\hat{\mathbf{r}}_{1} \cdot \hat{\mathbf{r}}_{j}}{r_{i}^{3} r_{1}^{8 / 3}}
$$

where $\hat{\mathbf{r}}_{j}$ is a unit vector in the direction of $\mathbf{r}_{j}$. Obviously also $I_{c}$ is local.

\section{B. Considerations involving arbitrary diagrams}

We can consider now arbitrary diagrams appearing in the Dyson equation. Every such diagram has a sequence of Green's functions connecting an entry at $\mathbf{r}$ to an exit at $\mathbf{r}^{\prime}$ via intermediate points $\mathbf{r}_{1}, \mathbf{r}_{2}, \ldots \mathbf{r}_{n}$, see Fig.2, panel (a). We call this sequence of Green's functions the "principal path" of the diagram. From vertices along this principal path there emanate either a double correlator or a subtree that begins with a Green's function that serves as its root.

Consider first the situation in which $r^{\prime} \leq r$. Our aim is to show that the main contribution to the diagram arises from the region of integration in which all the vertices are within the ball of locality of radius $O(r)$. Accordingly, we estimate first the mass operator $\Sigma$ in the case that all the vertices are within the ball of locality. Using Eq.(4.2) the evaluation of $\Sigma$ within the one-loop approximation is straightforward:

$$
\Sigma(r, r) \sim S_{2}(r) / r^{5}
$$

The two-loop diagrams contain two additional Green's functions which contribute a factor of $1 / r^{6}$, one correlator that contributes a factor of $S_{2}(r)$ [cf.(2.5)], two vertices that contribute a factor of $1 / r^{2}$, and two additional spacetime integrations which give a factor of $r^{6} \tau(r)^{2}$. Together all these additional factors give $S_{2}(r) \tau(r)^{2} / r^{2}$. Using the 
scaling relation (4.23) we conclude that this is $r^{0}$, and the two-loop diagrams are of the same order as the oneloop diagram. In fact, every order diagram in $\Sigma$ gives the same evaluation as (5.10). We remark that this is a common property of scale-invariant solutions in strong coupling systems.

Next consider a contribution in which the coordinates of all the vertices in the diagram except one, say at a point $r_{n}$, lie within a ball of radius $r$. This case can be dealt with using the analysis of sec.4.1: the situation locally must be one of the geometries (a), (b) or (c) in Fig.3. But we have shown that in all these cases the main contribution comes from the region $r_{n} \approx r$. In fact the evaluation of the contribution will be diminished by a factor of $\left(r / r_{n}\right)^{\alpha}$ where the exponent $\alpha$ takes the following values according to the local geometry:

$$
\alpha= \begin{cases}7 / 3 & (a) \\ 7 / 3 & (b) \\ 13 / 3 & (c)\end{cases}
$$

A situation in which a number of vertices go out of the ball of radius $r$ but do not have direct interactions between them is just a simple multiple of Eq.(5.11). Therefore the next situation to consider is that of a contribution with an arbitrary number of vertices outside the ball of radius $r$ with interactions. We take all of them to have $r_{n}$ of the same order, say of order $\tilde{r} \gg r$. Such a blob of vertices can be connected to the ball of radius $r$ via three legs again as in the situation (a), (b) and (c), or via a larger number of legs, see Fig. 1 . First consider the decoration of the uppermost vertex as in Fig. 1 a,b and c. The decorated vertex has two additional Green's functions, one additional correlator, and two space-time integrations. As we have learned such a combination scales like $r^{0}$. Notwithstanding, the estimates of the reduction in the importance of these contributions compared to (5.10) change from (5.11). The examples in Fig 4 a, $\mathrm{b}, \mathrm{c}$ are evaluated as

$$
\Sigma \sim\left\{S_{2}(r) / r^{5}\right\}(r / \tilde{r})^{2} .
$$

The reasons for this evaluation are as follows: (i) The evaluation of the decorated vertex is $1 / \tilde{r}$ (recall that the bare vertex is $\partial / \partial \tilde{r}$ which has the same evaluation); (ii) the Green's function connected via a straight line to the decorated vertex is evaluated as $r / \tilde{r}^{4}$; (iii) the space integration gives $\tilde{r}^{3}$. Lastly, the two additional legs in Fig.4, a have no $\tilde{r}$ dependence, and in toto we have (5.12). Note that in $5 \mathrm{~b}$ and $5 \mathrm{c}$ we have one and two Green's functions respectively. One could think that the time integrations over these Green's functions would introduce factors $\tau(\tilde{r})$ and $[\tau(\tilde{r})]^{2}$ respectively. This is not so; the time integration in the body of the diagram is restricted already by $\tau(r)$ because of the propagators in the body. Therefore the appropriate factors to use are $\tau(r)$ and $[\tau(r)]^{2}$ that do not change the dependence on $\tilde{r}$.

Decorating the vertex further (which is equivalent to looking at higher order contributions to the renormalized vertex) does not change the estimate (5.12). The scaling relation (4.37) protects the evaluation of the contribution. Therefore we consider now the situation in which the cluster of vertices at $\tilde{r}$ contains an additional vertex that does not decorate the bare vertex, and which comes with an additional leg that connects the blob at $r$ (Fig] $\mathrm{d}$ and e). An additional vertex is worth $1 / \tilde{r}$. The additional Green's function is worth $1 / \tilde{r}^{3}$, and the additional space integration brings in $\tilde{r}^{3}$. The additional time integral is irrelevant since it is restricted by the shorter times in the body of the diagram. Adding more legs connected to the blob always reduces the contribution further.

To complete the proof that the diagrams in the Dyson equation are dominated by their local contribution we now consider the situation that two blobs of vertices are located outside the ball of locality, say one blob at $\tilde{r}_{1}$ and $\tilde{r}_{2}$, see Fig 5 . There are two distinct possibilities: (i) the reduction factor depends on $\tilde{r}_{2}$ like some power of $\tilde{r}_{2}$ and (ii) the reduction factor is independent of $\tilde{r}_{2}$. In case (i) the power may be either positive or negative. If it is positive, then the main contribution comes from $\tilde{r}_{2} \sim \tilde{r}_{1}$, as shown in Fig. 5a. If the power is negative, the main contribution comes from $\tilde{r}_{2} \approx r$, as shown in Fig. 5ic. Both situations have one blob outside the ball of locality, and are covered by the discussion above. In case (ii) we will gain a $\operatorname{logarithm} \log \left(\tilde{r}_{1} / \tilde{r}_{2}\right)$ that will multiply the power $\left(r / \tilde{r}_{1}\right)^{\alpha}$ that was derived above for the single blob situation. This of course does not change the conclusion that the main contribution comes from the ball of locality.

The situation in which $r$ and $r^{\prime}$ are of different orders of magnitude poses the question whether the ball of locality is of radius $r$ or $r^{\prime}$. This question can be answered using the techniques developed above, but the natural framework to deal with this issue is to make use of the property of "rigidity" which will be defined in paper II of this series [5]. The result [5] is that the ball of locality is always determined by the first coordinate in the case of the Green's function $G\left(0 \mid \mathbf{r}, \mathbf{r}^{\prime}, t\right)$ and by the smaller coordinate in the case of the correlation function $F\left(0 \mid \mathbf{r}, \mathbf{r}^{\prime}, t\right)$.

\section{Locality with respect to small intermediate coordinates and small separation distances}

So far we have only considered the possibility that intermediate points $\mathbf{r}_{j}$ go out of the ball of locality, and we have shown that this diminishes drastically the contributions to our diagrams. It is known that this is the dangerous divergence that appears in the Eulerian perturbation methods. Nevertheless we discuss here for completeness the possibility that intermediate points $\mathbf{r}_{j}$ approach $\mathbf{r}_{0}$, or the origin of coordinates in our system that is centered at $\mathbf{r}_{0}$.

The discussion parallels to a large extent the steps that we took for the large distance case. Consider the situations of Fig. 3, but now since $r_{1}$ is the smallest coordinate 
in the group, one should invert the coordinate system. This is like looking at the figures upside down, and mirrored across a line parallel to the $\mathbf{r}$ axis. (Causality always forces $G\left(0 \mid \mathbf{r}, \mathbf{r}_{1}, t_{1}-t\right)$ to point from left to right in our coordinates where $t$ increases to the left). The main difference with the previous calculation can be seen right away, say in the situation (a):

$$
\begin{aligned}
(\mathrm{a}) & =\tau(r) \int_{0}^{r} r_{1}^{2} d r_{1} \frac{\partial}{\partial r} G\left(0 \mid \mathbf{r}, \mathbf{r}_{1}, 0^{+}\right) \\
& \times \frac{\partial}{\partial r_{1}}\left[F\left(0 \mid \mathbf{r}_{1}, \mathbf{r}_{2}, 0\right) F\left(0 \mid \mathbf{r}_{1}, \mathbf{r}_{3}, 0\right)\right]
\end{aligned}
$$

Since the leading contribution to $G\left(0 \mid \mathbf{r}, \mathbf{r}_{1}, 0^{+}\right)$, i.e. $1 / r_{1}^{3}$, disappears with the $\partial / \partial r$ differentiation, we take the next order which is $1 / r^{3}$ [cf. Eq. 4.6) and the next line]. This factor does not influence the $r_{1}$ integration, and we gain a factor of $r_{1}^{4}$ compared with the integration in the domain $(r, \infty)$. This difference now causes the integral to contribute at the upper limit, again at $r_{1} \approx r$. The same phenomenon recurs in all the relevant integrals, and we leave the completion of the proof, which is along the lines of the previous one, to the interested reader.

Lastly, we need to consider also the situation in which two (or more) coordinates approach each other, e.g $\left|\mathbf{r}_{i, j}\right|=\left|\mathbf{r}_{i}-\mathbf{r}_{j}\right| \ll r$. It is an immediate consequence of our choice of BL variables that nothing depends explicitly on this separation. Since we integrate over this separation from zero to $r$, the phase volume $r_{i, j}^{2} d r_{i, j}$ ensures that the main contribution comes from $\left|\mathbf{r}_{i, j}\right| \sim r$.

In summary, we have shown that the exact resummation of the Eulerian perturbation theory which results from the Belinicher-L'vov transformation leads to a new perturbation theory in which all the diagrams are local. We reiterate that "locality" here means that the major contribution comes from intermediate coordinates that are in a shell of radius $r$. The width of this shell is also of the order of $r$. This was proven in the last paragraph when we showed that the contributions from the center of the ball of locality with $r_{1} \ll r$ are also negligible. If we measure separation distances on a logarithmic scale, as is appropriate in a scale invariant situation, we can state that the major contribution to the diagrams come from the narrow shell of the ball of locality.

We can state these results in an intuitive fashion. With a perturbation at a point $\mathbf{r}^{\prime}$, from observing the differences of responses at points $\mathbf{r}$ and $\mathbf{r}_{0}$, we learn from our diagrammatic expansion that the coordinates of all the vertices [which designate the position of the hydrodynamic interactions $(\mathbf{u} \cdot \boldsymbol{\nabla}) \mathbf{u}]$ that appear in any order of the expansion must lie within a physical ball of fluid of radius $\left|\mathbf{r}-\mathbf{r}_{0}\right|$ in order to have a significant effect. Also the double correlator is affected by intermediate points in a finite ball of fluid of radius $\max \left(\left|\mathbf{r}-\mathbf{r}_{0}\right|,\left|\mathbf{r}^{\prime}-\mathbf{r}_{0}\right|\right)$. This is an analytic demonstration of the locality of the interactions between turbulent fluctuations in physical space.

\section{DISCUSSION: THE IMPLICATIONS FOR SCALING SOLUTIONS}

We can examine now the implications of the fact that, order by order, our diagrammatic expansion is local. First we show that K41 scaling is indeed a solution. Taking

$$
S_{2}(r) \sim r^{\zeta_{2}}, \quad \tau(r) \sim r^{z}
$$

we see that Eq.(4.37), which has been obtained solely from the property of locality, furnishes one scaling relation between $\zeta_{2}$ and $z$, i.e.

$$
\zeta_{2}+2 z=2 .
$$

We can easily find a second scaling relation by using our theory to calculate the 3 -point correlation function

$$
\begin{aligned}
& F_{\alpha \beta \gamma}\left(\mathbf{r}_{0} \mid \mathbf{r}_{1}, \mathbf{r}_{2}, \mathbf{r}_{3}, t_{1}, t_{2}, t_{3}\right) \\
= & \left\langle w_{\alpha}\left(\mathbf{r}_{0} \mid \mathbf{r}_{1}, t_{1}\right) w_{\beta}\left(\mathbf{r}_{0} \mid \mathbf{r}_{2}, t_{2}\right) w_{\gamma}\left(\mathbf{r}_{0} \mid \mathbf{r}_{3}, t_{3}\right)\right\rangle .
\end{aligned}
$$

The simultaneous correlation $F_{\alpha \beta \gamma}\left(\mathbf{r}_{0} \mid \mathbf{r}, \mathbf{r}, \mathbf{r}, t, t, t\right)$ is identical to the 3-rd order structure function of the Eulerian field, cf. Eq.(2.5), and it is well known that for small viscosity it scales (in the inertial range) like $r$. The 3point correlation function has a totally analogous theory to the one described above for the 2-point function, and a typical first order diagram for this quantity is shown in Fig.6. By repeating the analysis that led to the proof of locality we see that the main contribution to the integral $\int d \mathbf{r}_{1} d t_{1}$ Fig. 7a comes from the region $r_{1} \sim r$ and $t_{1} \sim \tau(r)$. Consequently we can evaluate $S_{3}(r)$ in the first order as

$$
S_{3}(r) \sim \tau(r)\left[S_{2}(r)\right]^{2} / r .
$$

The next order contributions to $F_{\alpha \beta \gamma}$ are nothing but the renormalization of the bare vertex, see for example Fig. 6b. One can prove following the ideas of Sec. 4 that all these diagrams are local. The property of locality and the scaling relation (4.37) or equivalently (6.2) imply that all the n'th order diagrams have the same evaluation as the first order diagram (6.4). Since $S_{3}(r) \sim r$, we find a second scaling relation

$$
\left[S_{2}(r)\right]^{2} \tau(r) \sim r^{2}
$$

In terms of the scaling exponents this is

$$
2 \zeta_{2}+z=2 \text {. }
$$

The solution of 6.2 and $(6.5)$ is $\zeta_{2}=2 / 3, \mathrm{z}=2 / 3$, which are the standard K41 exponents.

The usual mechanisms to renormalize exponents are connected to the divergence of the integrals appearing in higher order diagrams. The cutoff lengths that are needed to control the divergence can be used to form dimensionless corrections to the scaling functions, and thus to renormalize the exponents. Since our diagrams 
are local, there are no typical scales which appear in this way, and we do not have a simple way to renormalize the scaling exponents of the structure functions. Indeed, if there is a renormalization of the exponents, it must stem from nonperturbative effects. The study of these effects and the elucidation of multiscaling is the subject of papers II and III in this series.

\section{ACKNOWLEDGMENTS}

The content of this paper owes a great deal to some persistent criticism of the $\mathbf{k}, \omega$ presentation by U. Frisch and R.H. Kraichnan. We are particularly grateful to R. H. Kraichnan for some deep discussions on these issues, and to Adrienne Fairhall, Daniel Segel and Stefan Thomae for important critical comments on the manuscript. This work has been supported in part by the The Naftali and Anna Backenroth-Bronicki Fund for Research in Chaos and Complexity, Minerva Foundation, Munich, Germany, the German Israeli Foundation and the Basic Research Fund of the Israeli Academy of Sciences. IP acknowledges the hospitality of the MittagLeffler Institute, Stockhlom, where a portion of this manuscript was written.

\section{APPENDIX A: RELATION BETWEEN THE CORRELATION FUNCTIONS BEFORE AND AFTER THE BL TRANSFORMATION}

In this Appendix (which follows [28]) we examine the relation between the BL transformed correlation functions and the correlation functions of the Eulerian fields. The aim of this appendix is to clarify how the stationarity and homogeneity of the original fields are reflected in the properties of the transformed fields. We introduced the BL velocity $\mathbf{v}$ by the expression 2.2$): \mathbf{v}(t, \mathbf{r})=\mathbf{u}(t, \mathbf{r}+\boldsymbol{\rho})$ where $\mathbf{u}$ is the Eulerian velocity and $\boldsymbol{\rho}(t)$ introduced by (2.3) is governed by the equation

$$
\partial \boldsymbol{\rho} / \partial t=\mathbf{u}\left(t, \mathbf{r}_{0}+\boldsymbol{\rho}\right)=\mathbf{v}\left(t, \mathbf{r}_{0}\right), \quad \boldsymbol{\rho}\left(t=t_{0}\right)=\mathbf{0}
$$

Here $t_{0}$ is a marked time and $\mathbf{r}_{0}$ is a marked point which can be chosen arbitrary. Equation (A1) describes the Lagrangian trajectory $\mathbf{r}_{0}+\boldsymbol{\rho}$ of the liquid point starting from the point $\mathbf{r}_{0}$ at $t=t_{0}$.

Let us introduce a designation $\Psi$ for the product of the $\mathrm{BL}$ velocities taken in different spatial-time points,

$$
\begin{aligned}
& \Psi\{\boldsymbol{\rho}\}=v\left(t_{1}, \mathbf{r}_{1}\right) v\left(t_{2}, \mathbf{r}_{2}\right) \ldots v\left(t_{n}, \mathbf{r}_{n}\right) \\
= & u\left[t_{1}, \mathbf{r}_{1}+\boldsymbol{\rho}\left(t_{1}\right)\right] u\left[t_{2}, \mathbf{r}_{1}+\boldsymbol{\rho}\left(t_{2}\right)\right] \ldots u\left[t_{n}, \mathbf{r}_{n}+\boldsymbol{\rho}\left(t_{n}\right)\right] .
\end{aligned}
$$

Next we transform the expression for the different-time correlation function of BL velocities, $\langle\Psi\{\boldsymbol{\rho}\}\rangle$, into the form which is convenient for utilizing the relation between $\mathbf{u}$ and $\mathbf{v}$. For this goal we will treat $\boldsymbol{\rho}$ as an independent field taking into account the equation (A1) via the corresponding functional $\delta$-function. Namely $\langle\Psi\{\boldsymbol{\rho}\}\rangle$ can be rewritten as

$$
\int \mathcal{D} \rho\left\langle\delta\left\{\partial \boldsymbol{\rho} / \partial t-\mathbf{u}\left(t, \mathbf{r}_{0}+\boldsymbol{\rho}\right)\right\} \Psi\{\boldsymbol{\rho}\}\right\rangle,
$$

where $\int \mathcal{D} \rho$ means the path integral over all functions $\boldsymbol{\rho}(t)$. The functional $\delta$-function, $\delta\{\ldots\}$ can be represented in the exponential form with the help of auxiliary field $\boldsymbol{\beta}(t)$. Then we find

$$
\begin{array}{r}
\langle\Psi\{\boldsymbol{\rho}\}\rangle=\int \mathcal{D} \rho \mathcal{D} \beta \exp \left(i \int d t \boldsymbol{\beta} \partial \boldsymbol{\rho} / \partial t\right) \\
\times\left\langle\exp \left(-i \int d t \boldsymbol{\beta} \mathbf{u}\left(t, \mathbf{r}_{0}+\boldsymbol{\rho}\right)\right) \Psi\{\boldsymbol{\rho}\}\right\rangle .
\end{array}
$$

In the above formal transformations we omitted the functional determinant arising at passing to integration over $\rho, \beta$. To recognize the value of the determinant we should first discretize the problem introducing instead of (A1) a discrete scheme, e.g.

$$
\begin{aligned}
& \boldsymbol{\rho}\left(t_{n+1}\right)-\boldsymbol{\rho}\left(t_{n}\right)-\mathbf{u}\left(t, \mathbf{r}_{0}+\boldsymbol{\rho}\left(t_{n}\right)\right) \Delta t=0, \\
& t_{n}=t_{0}+n \Delta t .
\end{aligned}
$$

Now the functional $\delta$-function in $\mathrm{A} 3$ is the product of usual $\delta$-functions with arguments defined by the left-hand sides of the equation $\mathrm{A} 5$, the product being taken for all numbers $n \neq 0$ ( since in accordance with (A1) $\boldsymbol{\rho}\left(t_{0}\right)=\mathbf{0}$ ). The functional integration in (A3) is now the product of integrations over $\rho\left(t_{n}\right), n \neq 0$. We see that for $n>0$ the consequent integration over $\boldsymbol{\rho}\left(t_{n}\right)$ actually gives unity since

$$
\int d \boldsymbol{\rho}\left(t_{n+1}\right) \delta\left(\boldsymbol{\rho}\left(t_{n+1}\right)-\boldsymbol{\rho}\left(t_{n}\right)-\ldots\right)=1 .
$$

But when we go over $n$ in the negative direction the integrals over $\boldsymbol{\rho}\left(t_{n}\right)$ produce nontrivial factors associated with a dependence of $\mathbf{u}$ on $\boldsymbol{\rho}$. The product of these factors is just the functional determinant noted above.

Usually this problem is avoided by putting $t_{0}$ into $-\infty$, after that the actual region of $t$ produces the unitary determinant. It is possible to do only if we deal with a situation as a stationary one which is not sensitive to initial conditions. Just this reason explains that the corresponding functional determinant can be regarded to be equal to unity in the path integral formulation of the Wyld-Martin-Rose-Siggia technique. But our problem is in proving time homogeneity of BL correlation functions, therefore $t_{0}$ is a finite time and we should consider times $t$ both before and after $t_{0}$. Fortunately in our case the incompressibility condition ensures the unitary of the determinant. The point is that for small $\Delta t$ in the approximation needed for us the integrals

$$
\int d \boldsymbol{\rho}\left(t_{n-1}\right) \delta\left(\boldsymbol{\rho}\left(t_{n}\right)-\boldsymbol{\rho}\left(t_{n-1}\right)-\ldots\right)=1-\Delta t \boldsymbol{\nabla} \mathbf{u}
$$


are equal to unity due to the incompressibility condition. Thus we see that the condition is of the crucial importance for the Belinicher-L'vov scheme.

Taking into account the definition (A1) we conclude that the expression in the angle brackets in (A4) is an average of a combination of Eulerian velocities $\mathbf{u}(t, \mathbf{r}+\boldsymbol{\rho}(t))$ with a function $\boldsymbol{\rho}(t)$. This average is obviously translationally invariant. It allows one to shift all space arguments at a constant vector $\mathbf{R}$. Let us choose $\mathbf{R}=\boldsymbol{\rho}(\tau)$ where $\tau$ is some time. Then (A4) acquires the form

$$
\begin{gathered}
\langle\Psi\{\boldsymbol{\rho}\}\rangle=\int \mathcal{D} \rho^{\prime} \mathcal{D} \beta \exp \left(i \int d t \boldsymbol{\beta} \partial \boldsymbol{\rho}^{\prime} / \partial t\right) \\
\times\left\langle\exp \left(-i \int d t \boldsymbol{\beta} \mathbf{u}\left(t, \mathbf{r}_{0}+\boldsymbol{\rho}^{\prime}\right)\right) \Psi\left\{\boldsymbol{\rho}^{\prime}\right\}\right\rangle,
\end{gathered}
$$

where $\boldsymbol{\rho}^{\prime}(t)=\boldsymbol{\rho}(t)-\boldsymbol{\rho}(\tau)$. Note that $\boldsymbol{\rho}^{\prime}(\tau)=\mathbf{0}$. Integration over $\rho, \beta$ in (A6) gives

$$
\langle\Psi\{\boldsymbol{\rho}\}\rangle=\left\langle\Psi\left\{\boldsymbol{\rho}^{\prime}\right\}\right\rangle,
$$

where $\boldsymbol{\rho}^{\prime}(t)$ is governed by the same equation (A1) as $\boldsymbol{\rho}$ but with the initial condition $\boldsymbol{\rho}^{\prime}(\tau)=\mathbf{0}$.

The relation (A7) means that $\mathrm{BL}$ correlation functions are stationary in time since they do not actually depend on the marked time $\tau$. Using (A7) one can easily prove the coincidence of simultaneous correlation functions of BL and Eulerian velocities. Namely, taking in (A2) all times $t_{1}, t_{2}, \ldots$ to be equal to $t$ and choosing in (A7) $\tau=t$ we immediately find

$$
\begin{aligned}
& \left\langle v\left(t, \mathbf{r}_{1}\right) v\left(t, \mathbf{r}_{2}\right) \ldots v\left(t, \mathbf{r}_{n}\right)\right\rangle \\
= & \left\langle u\left(t, \mathbf{r}_{1}\right) u\left(t, \mathbf{r}_{2}\right) \ldots u\left(t, \mathbf{r}_{n}\right)\right\rangle,
\end{aligned}
$$

since $\boldsymbol{\rho}^{\prime}(t)=0$ in this case. Although we examined correlation functions of BL velocities, all arguments presented above can be applied to arbitrary fields e.g. pumping forces. It implies e.g. that BL Green's functions are stationary in time and that their simultaneous values coincide with ones of Eulerian Green's functions.

* L'vov's e-mail: fnlvov@wis.weizmann.ac.il.

$\dagger$ Procaccia's e-mail: cfprocac@weizmann.weizmann.ac.il.

[1] A.S. Monin and A. M. Yaglom, Statistical Fluid Mechanics (MIT press, 1975)

[2] K.R. Sreenivasan, Annu. Rev. Fluid Mech. 23,539 (1991).

[3] M. Nelkin Advan. in Phys. 43 143-181 (1994).

[4] V. I. Belinicher and V. S. L'vov. A scale-invariant theory of fully developed hydrodynamic turbulence. Sov. Phys. JETP, 66:303-313, 1987.

[5] V. S. L'vov and I. Procaccia. Exact resummations in the theory of hydrodynamic turbulence. II. A ladder to anomalous scaling. Phys. Rev. E, 1995. Next paper in this issue.
[6] V.S. L'vov and I. Procaccia, Exact resummations in the theory of hydrodynamic turbulence. III. Scenarios for Anomalous Scaling and Intermittency, Submitted to Phys.Rev.E

[7] V. S. L'vov and V. V. Lebedev. Anomalous scaling in Kolmogorov 1941 turbulence. Europhys. Lett., 29 681-686 (1995).

[8] V. V. Lebedev and V. S. L'vov. Scaling of correlation functions of velocity gradients in hydrodynamic turbulence. JETP Letters, 59:577-583, 1994.

[9] V. S. L'vov and I. Procacia. "Intermittency" in turbulence as intermediate asymptotics to Kolmogorov'41 scaling. Phys. Rev. Lett., 74, 2690 (1995).

[10] H. W. Wyld. Formulation of the theory of turbulence in an incompressible fluid. Ann. Phys., 14:143-165, 1961.

[11] P. C. Martin, E. D. Siggia, and H. A. Rose. Statistical dynamics of classical systems. Phys. Rev. A, 8(1):423-437, July 1973.

[12] R. H. Kraichnan. Eulerian and Lagrangian renormalization in turbulence theory. J. Fluid Mech., 83(2):349-374, 1977.

[13] C. DeDominics. J. Physique (Paris), 37:C1-247, 1976.

[14] H.K. Jansen. Z. Phys. B, 23:377, 1976.

[15] V. S. L'vov and I. Procaccia. Exact resummations in the theory of hydrodynamic turbulence. 0 Line-resummed diagrammatic perturbation approach. In F. David and P. Ginsparg, editors, Lecture Notes of the Les Houches 1994 Summer School, 1995. In press.

[16] L. Richardson. Weather Prediction by Numeric Process. Cambridge Univ. Press, Cambridge, 1922.

[17] Lewis F. Richardson. Atmospheric diffusion shown on a distance neighbor graph. Proc. R. Soc. Lond. A, 110:709737, 1926.

[18] A. N. Kolmogorov. The local structure of turbulence in incompressible fluid for very large Reynolds numbers. C. R. (Doklady) Acad. Sc. URSS, 30:9-13, 1941.

[19] R. H. Kraichnan, 1994. Private communication.

[20] G. Stolovitzky and K.R. Sreenivasan. Scaling of structure functions. Phys. Rev. E, 48(1):R33-R36, 1993.

[21] R. Benzi, S. Ciliberto, R. Tripiccione, C. Baudet, F. Massaioli, and S. Succi. Extended self-similarity in turbulent flows. Phys. Rev. E, 48(1):R29-R32, July 1993.

[22] C-Y. Mou and P. B. Weichman. Multicomponent turbulence, the spherical limit, and non-Kolmogorov spectra. CalTex, , 1994. Preprint.

[23] V. S. L'vov, I. Procaccia, and A. Fairhall. Anomalous scaling in fluid dynamics: The case of passive scalar. Phys. Rev. E, 50(6):4684-4704, December 1994.

[24] V. S. L'vov and I. Procaccia. Correlator of velocity differences and energy dissipation as element in the subcritical scenario for non-Kolmogorov scaling in turbulence. Europhys. Lett., 29 (4) 291-296, 1995.

[25] V. S. L'vov and V. V. Lebedev. Exact relations in the theory of fully developed hydrodynamic turbulence. Phys. Rev. E, 47(4):1794-1802, 1993.

[26] E. Kats and V. Lebedev. Fluctuation effect in the dynamic of liquid crystals. Springer Verlag, Heidelberg, 1993.

[27] A. A. Praskovsky and S. Oncley. Correlators of velocity differences and energy dissipation at very high Reynolds numbers. Europhys. Lett., 29 (9) 635-640 1994.

[28] V. V. Lebedev and V. S. L'vov, e-print in Los-Alamos e- 
board No.9410003 (1994) chao-dyn@xyz.lanl.gov

$$
\begin{aligned}
& \mathrm{W} \sim m, \mathrm{w}_{\mathrm{O}} \sim \sim \text {, } \\
& \text { * } \Gamma: \sim-\tau_{2} \\
& \mathrm{~F} \sim \text { onsu, } \mathrm{F}_{\mathrm{o}} \sim \cos r \text {, } \\
& \mathrm{G} \sim \operatorname{nr}, \mathrm{G}_{0} \sim \operatorname{rr} \text {. }
\end{aligned}
$$

FIG. 1. Graphical notation for the perturbation expansion. The symbol used are the following: short wavy lines stand for the fluid velocity (think about waves). A thin wavy line stands for $\mathbf{u}_{0}$, whereas a bold wavy line represents the full solution $\mathbf{u}(\mathbf{r}, t)$. A straight line stands for the field $\mathbf{p}(\mathbf{r}, t)$ that appear in the functional integral approach. The Green's function, which is the response in the velocity to some force is made of a short wavy line and a short straight line representing the force. Again this symbol appears in thin and bold variants. the former stands for the bare Green's function (2.20), and the bold for the dressed Green's function (2.18) The vertex (3.1) is a fat dot with three tails. One straight tail belongs to the Green's function, and two wavy tails stand for velocities. A long wavy line will represent correlation functions of velocities. Again, the thin and bold variants are bare and dressed correlators respectively.

(a)

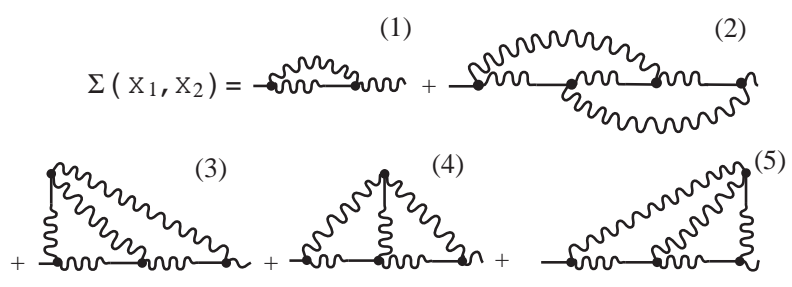

(b)

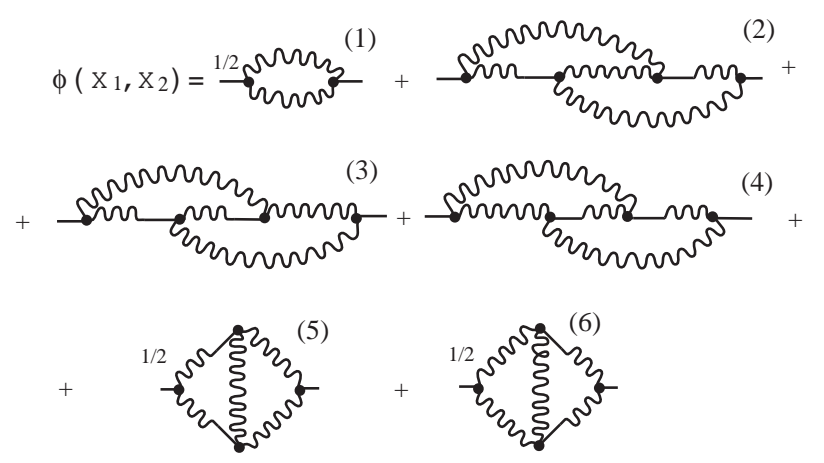

FIG. 2. Diagrammatic representation of the renormalized series expansion for the mass operators. (a) The mass operator $\Sigma$ of the Dyson equation (2.23) and (b) the mass operator $\Phi$ of the Wyld equation (2.24).

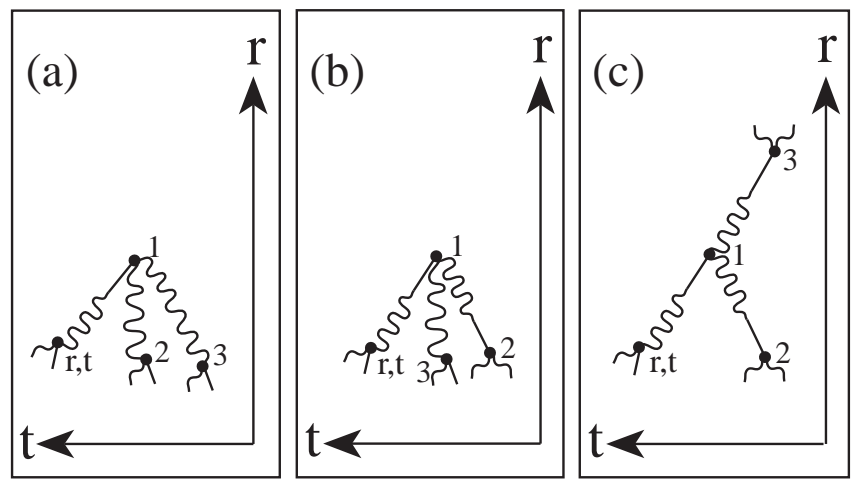

FIG. 3. Three different arrangements of four adjacent vertices in a fragment of a diagram, drawn in $\mathbf{r}, t$ space.
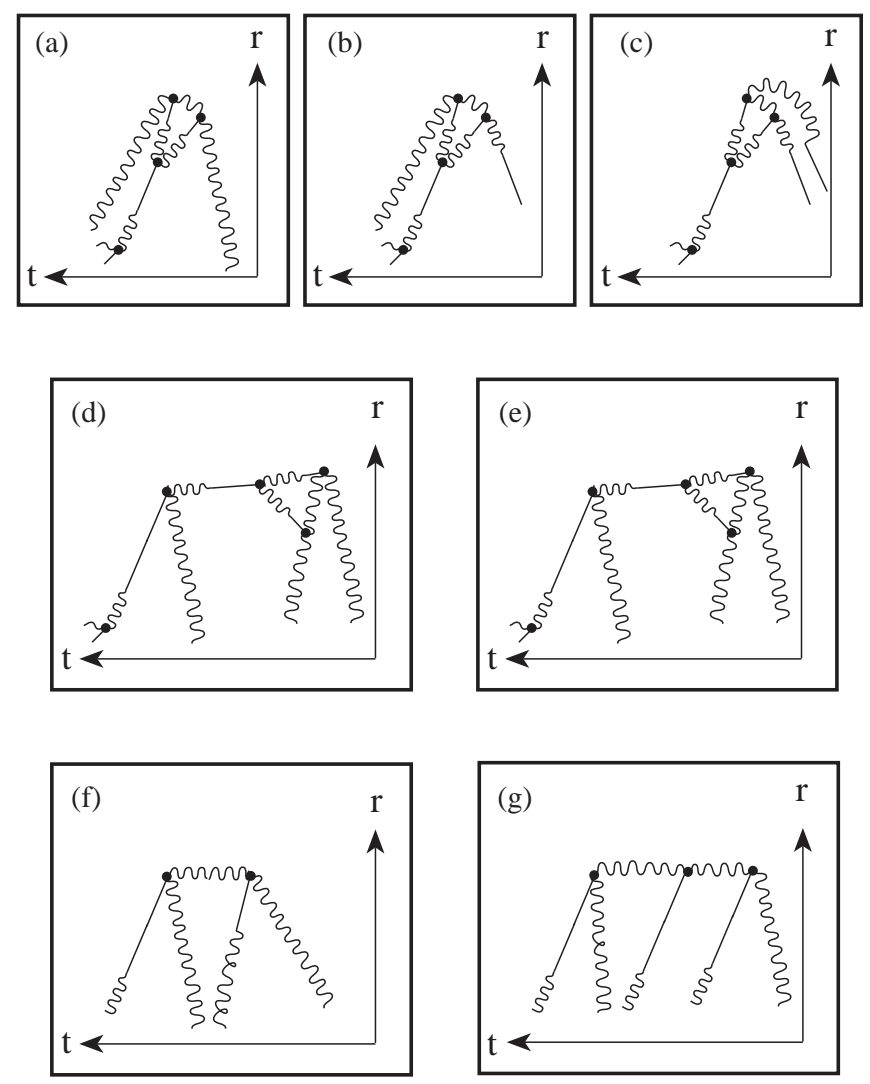

FIG. 4. Fragments of diagrams which are used in the proof of locality. 

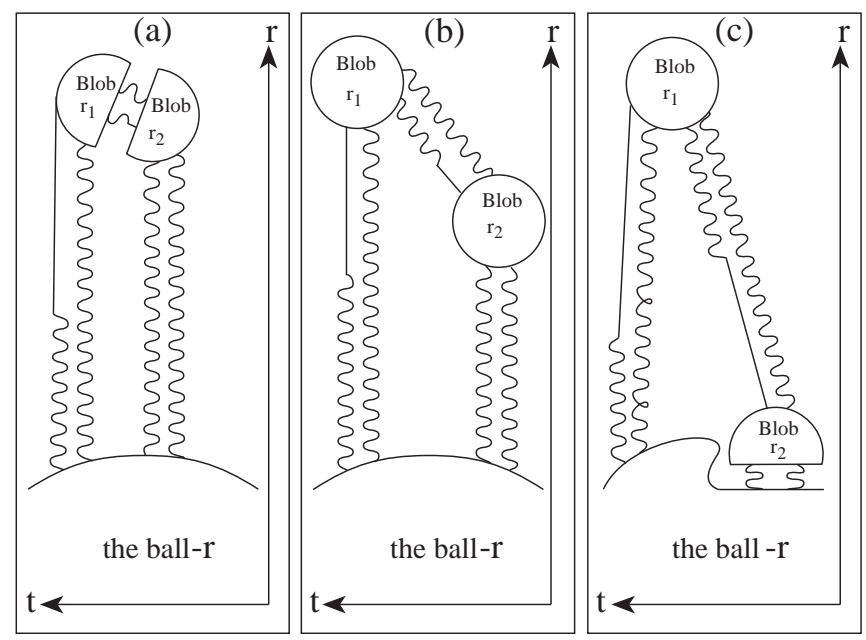

FIG. 5. Fragments of diagrams which are used in the global considerations in the proof of locality.
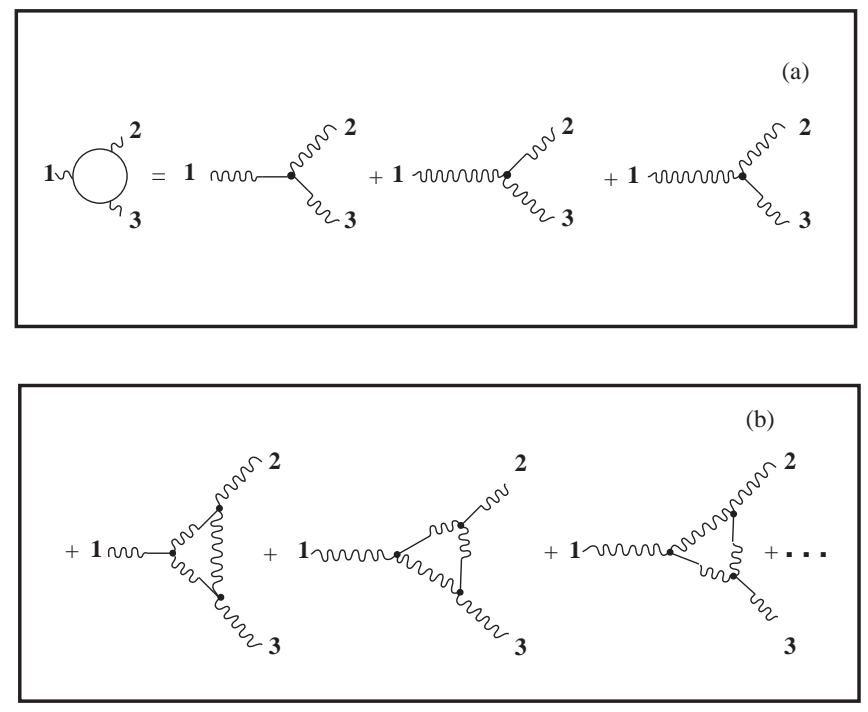

FIG. 6. Diagrams in the expansion for the triple correlation function. (a) All the first order contributions, (b) some of the third order contributions. 